\title{
Profit Maximization of a Power Plant
}

Kragelund, Martin Nygaard; Leth, John-Josef; Wisniewski, Rafal; Jönsson, Ulf

Published in:

European Journal of Control

DOI (link to publication from Publisher):

10.3166/EJC.18.38-54

Publication date:

2012

\section{Document Version}

Accepted author manuscript, peer reviewed version

Link to publication from Aalborg University

Citation for published version (APA):

Kragelund, M. N., Leth, J-J., Wisniewski, R., \& Jönsson, U. (2012). Profit Maximization of a Power Plant. European Journal of Control, 18(1), 38-54. https://doi.org/10.3166/EJC.18.38-54

\section{General rights}

Copyright and moral rights for the publications made accessible in the public portal are retained by the authors and/or other copyright owners and it is a condition of accessing publications that users recognise and abide by the legal requirements associated with these rights.

- Users may download and print one copy of any publication from the public portal for the purpose of private study or research.

- You may not further distribute the material or use it for any profit-making activity or commercial gain

- You may freely distribute the URL identifying the publication in the public portal -

Take down policy

If you believe that this document breaches copyright please contact us at vbn@aub.aau.dk providing details, and we will remove access to the work immediately and investigate your claim. 


\title{
Profit Maximization of a Power Plant
}

\author{
Martin Kragelund, John Leth, Rafał Wisniewski, and Ulf Jönsson
}

\begin{abstract}
This paper addresses the problem of profit maximization of a power plant by utilizing three different fuel systems in an optimal manner. Pontryagin's maximum principle is used to derive properties of the optimal control strategy. These properties give rise to a switching function. Subsequently, certain heuristics are introduced and used in combination with discrete optimization to obtain an initial trajectory of the switching function. An iterative procedure is proposed, which uses the initial trajectory for the computation of the optimal control strategy. The control strategy derived is a combination of a state feedback and time-varying feedforward term. Its performance is tested against input noise.
\end{abstract}

\section{INTRODUCTION}

The economic perspective is rarely considered when developing control structures and strategies for process control systems. Indeed, requirements are most frequently imposed on disturbance rejection, pole placement or other well known system theoretic properties. Nonetheless, the economics of control has gained some focus for example the selection of sensor and actuator [15] and the design of controller structure [16]. Furthermore, optimal steady state operations have been studied [3].

In [8], the hydro power production in Norway is considered by maximizing the profit of a hydro plant such that the production commitment of the current day is fulfilled. The electrical market is considered in the optimization and planning of the production for which stochastic programming is used.

The work in this paper is similar to the work in [8] as profit maximization in electricity production is considered. However, a traditional power plant will be considered, and optimal control will be used for the profit optimization [19], [21].

A problem of the optimal operation of a power plant in a liberal electricity market is a subject of [20]. In this work, two types of power plants are considered: a hydroelectric and a thermal power plant. The main focus is on the dynamic modeling of electricity-production and the price of electricity. Both of them are described

This work is supported by The Danish Research Council for Technology and Production Sciences via Plug and Play Process Control and Hybrid Systems.

The authors would like to thank DONG Energy and in particular Kristian Edlund for their support during the writing of this paper.

The first, second, and third authors are all with the Department of Electronic Systems, Aalborg University, Fredrik Bajers Vej 7C, 9220 Aalborg $\varnothing$, Denmark \{mkr, jjl, raf\}@es.aau.dk.

The fourth author is with Department of Mathematics, Royal Institute of Technology, SE 100-44 Stockholm, Sweden ulfj@math.kth.se by random processes. As a consequence, the suggested approach to the optimal operation of a power plant is stochastic optimization, which is formulated in terms of nonlinear partial-integro-differential equations. To solve them, the authors use a finite difference scheme.

In like manner, [17] addresses the problem of balancing the power on electricity market consisting of wind energy and hydropower. Also in this work, the demand for balancing power and the electricity price are described by stochastic models. Subsequently, the hydropower-scheduling of trading decisions are formulated as a stochastic optimization problem. To solve it, the stochastic variables are approximated by a finite set of scenarios, so called scenario-trees. Afterward, the optimization problem is solved by means of stochastic programming.

A power plant capable of using a number of different fuels is considered in this paper. The fuels of interest are coal, gas, and oil. They certainly have certain advantages and disadvantages, e.g., coal is an inexpensive fuel, but it is difficult to control. The objective of this work is to maximize the profit of the power plant when following a predefined production reference.

The problem stated above has been discussed in [14], [12], [13], and [10]. In particular, the formulation in [10] which includes plant dynamics gives the basis of this work. In the previous works, a function for the instantaneous profit flow has been determined, which includes time-varying measures of business objectives and time varying price data obtained from Nord $\mathrm{Pool}^{1}$. The objective is to maximize the integral of the instantaneous profit flow over time, i.e., maximizing the profit of the company. The requirement of following a predefined reference has been formulated as a side-constraint in the optimization, which has been solved in discrete time. Whereas, in this work the tracking will be included as a penalty term in the objective function. This yields a simpler problem, which substantially reduces the computation time.

In this work, a continuous control strategy is given that maximizes the profit of a power plant. The strategy is obtained by using Pontryagin's maximum principle to devise some properties of the optimal control input. The optimal solution consists primarily of singular arcs which is known to make the optimal control problem more difficult to solve numerically. In this work, we propose an approach that results in a combined feedback and feed-

\footnotetext{
${ }^{1}$ Nord Pool is the Nordic electrical market, where power contracts are traded.
} 
forward solution, which yields a profit $30 \%$ greater than using an input signal obtained by discrete optimization. Feedback schemes for solving singular control problems have been proposed previously [22].

We anticipate the results in this paper are of interest in a model predictive control (MPC) context. Indeed, if the optimal solution is known to consist of singular arcs then this information should be used in the development of an optimal algorithm. By computing the feedback law that generates the singular arcs better performance and lower sampling rate may be achieved. This should be particularly useful when the model and the data is provided in continuous time over a large time horizon.

\section{A. Outline}

A model of the plant considered in this work is presented in Section II. Furthermore, the models of the business objectives and optimization problem are presented there. In Section III, Pontryagin's maximum principle is applied to the optimization problem and some properties of the optimal input are derived. As the optimal input is dependent on an unknown switching function, the optimization problem is converted to discrete time, and subsequently, an estimate of the switching function is computed. This procedure is carried out in Section IV. The switching function is applied to the optimal input strategy in Section V, and the resulting profit is compared to what was possible with the discrete optimization. In Section VI, the control strategy is evaluated when input noise is present, and finally, in Section VII, a discussion of the results is given. Furthermore, two appendices are included where the optimal continuous control strategy and a discrete version of the objective function are given.

\section{Problem Formulation}

In this section, the models from previous work will be recalled, and the optimization problem will be presented. First, an introduction to the considered plant is given.

The problem considered in this work is based on a coal fired boiler in power plant, which is depicted in Figure 1, and consists of the following components:

Coal mills: The coal mills grind the coal to small dust particles which burn quickly and efficiently. However, it is difficult to control the amount of dust the coal mills deliver as it is not possible to measure the dust flow into the furnace.

Furnace: The furnace is a module where the coal dust (or other fuels) is burned; thereby, heat is delivered to the boiler.

Evaporator: The evaporator is fed with water, which is evaporated under high pressure by the heat from the burners.

Superheater: The superheater (super) heats the steam from the evaporator.

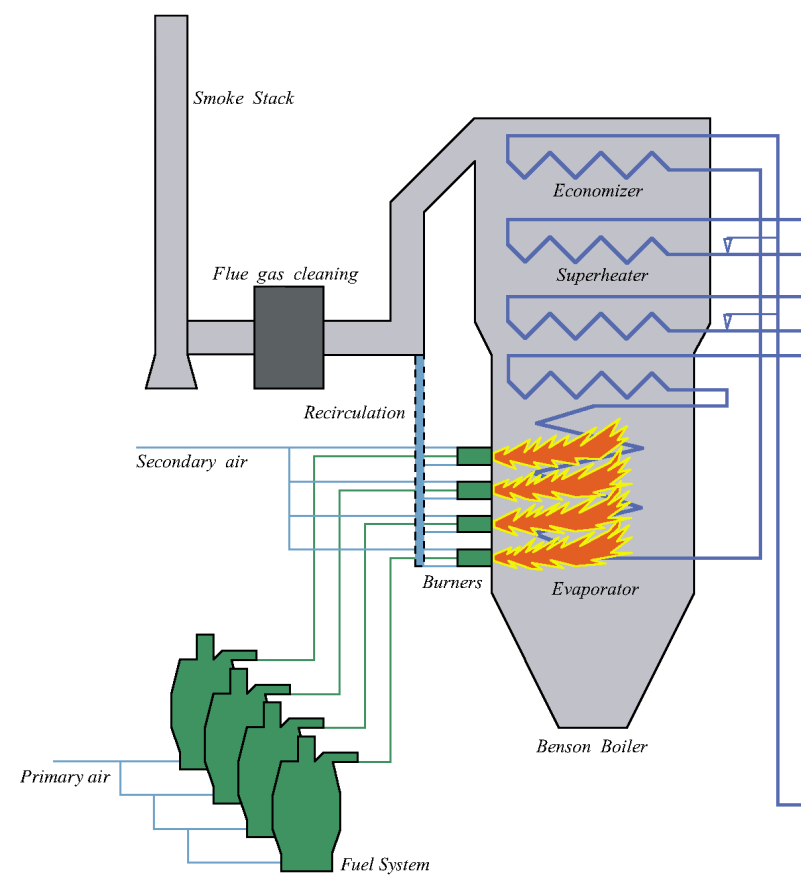

Fig. 1. Benson boiler model. The fuel system consists of the coal mills and a transport system to the furnace. Here the coal is burned in the burners and the heat is exchanged to water in the evaporator.

Economizer: The economizer uses some of the remaining heat in the flue gas to preheat the feed water before it enters the evaporator.

The illustrated model does not depict the flue gas cleaning and smoke stack and the conversion from steam power to electrical power is also left out. It is assumed that when the plant is operating at full load the electrical power produced amounts to $400 M W$. Furthermore, the power plant is in this work augmented with two additional fuel systems: gas and oil.

The three different fuels have certain advantages and disadvantages, e.g., gas is easy to control, but is an expensive fuel. Some of the characteristics of the different fuels are:

Coal is advantageous when considering the price per stored energy; however, it is difficult to control as unmeasurable fluctuations in the coal flow are introduced when the coal is ground to coal dust in the coal mill. This implies that changing the operating point of the system should be done slowly. Furthermore, the coal mills use some electrical energy to grind the coal, which needs to be considered.

Gas is more expensive than coal and energy is not converted to steam as efficiently with gas as with coal due to the layout of the chosen boiler. However, gas arrives at the power plant under high pressure, which is lowered using a turbine generating electrical energy. Furthermore, gas is much easier to control as it is possible to measure the flow. 
Oil is, with the current market prices, the most expensive of the three fuels and has to be preheated before entering the boiler. This process demands energy itself. Nevertheless, oil is considered in this work as it is possible to measure the oil flow into the boiler and this makes it easy to control. Furthermore, oil is present in most existing coal fired plants as oil is used to start up the plant.

In this work, it is assumed that the plant is controlled. Therefore, linear dynamics are sufficient to model it [7]. Indeed, it is shown in [7] that the change in the produced electricity caused by changing the fuel flow can be captured by third order dynamics. In the following, it is assumed that the fuel flow reference (in $\mathrm{kg} / \mathrm{s}$ ) of coal, gas, and oil is the input to the system, i.e., $\boldsymbol{u}=\left(u_{c}, u_{g}, u_{o}\right)$ is a vector of the coal, gas, and oil flow references respectively. The state vector consists of the actual flow of the different fuels and their first and second derivative, i.e., $\boldsymbol{z}_{c}=\left(z_{1}, z_{2}, z_{3}\right)$ is the coal flow into the boiler and its first and second derivative. Similarly, for the gas and oil systems $\boldsymbol{z}_{g}=\left(z_{4}, z_{5}, z_{6}\right)$ and $\boldsymbol{z}_{o}=\left(z_{7}, z_{8}, z_{9}\right)$. Therefore, the full state vector $\boldsymbol{z}$ is given by $\boldsymbol{z}=\left(\boldsymbol{z}_{c}, \boldsymbol{z}_{g}, \boldsymbol{z}_{o}\right)$. Furthermore, the complete dynamics of the three different fuel systems considered in this work is given by

$$
\begin{aligned}
\dot{\boldsymbol{z}}(t) & =\boldsymbol{A} \boldsymbol{z}(t)+\boldsymbol{B} \boldsymbol{u}(t) \\
\boldsymbol{x}(t) & =\boldsymbol{C} \boldsymbol{z}(t),
\end{aligned}
$$

where

$$
\begin{aligned}
& \boldsymbol{A}=\left[\begin{array}{ccc}
\boldsymbol{A}_{c} & \mathbf{0}_{3 x 3} & \mathbf{0}_{3 x 3} \\
\mathbf{0}_{3 x 3} & \boldsymbol{A}_{g} & \mathbf{0}_{3 x 3} \\
\mathbf{0}_{3 x 3} & \mathbf{0}_{3 x 3} & \boldsymbol{A}_{o}
\end{array}\right], \boldsymbol{A}_{i}=\left[\begin{array}{ccc}
0 & 1 & 0 \\
0 & 0 & 1 \\
h_{i_{1}} & h_{i_{2}} & h_{i_{3}}
\end{array}\right], \\
& \boldsymbol{B}=\left[\begin{array}{ccc}
\boldsymbol{B}_{c} & \mathbf{0}_{3 x 1} & \mathbf{0}_{3 x 1} \\
\mathbf{0}_{3 x 1} & \boldsymbol{B}_{g} & \mathbf{0}_{3 x 1} \\
\mathbf{0}_{3 x 1} & \mathbf{0}_{3 x 1} & \boldsymbol{B}_{o}
\end{array}\right], \boldsymbol{B}_{i}=\left[\begin{array}{c}
0 \\
0 \\
h_{i_{0}}
\end{array}\right], \\
& \boldsymbol{C}=\left[\begin{array}{ccc}
\boldsymbol{C}_{1} & \mathbf{0}_{1 x 3} & \mathbf{0}_{1 x 3} \\
\mathbf{0}_{1 x 3} & \boldsymbol{C}_{1} & \mathbf{0}_{1 x 3} \\
\mathbf{0}_{1 x 3} & \mathbf{0}_{1 x 3} & \boldsymbol{C}_{1}
\end{array}\right], \boldsymbol{C}_{1}=\left[\begin{array}{lll}
1 & 0 & 0
\end{array}\right],
\end{aligned}
$$

and $h_{i_{j}}, i \in \mathcal{I}=\{c, g, o\}$, are constants describing the dynamics of the three fuel systems, which are obtained from transfer functions of the form

$$
H_{i}(s)=\frac{1}{\left(\tau_{i} s+1\right)^{3}},
$$

where $\tau_{i}, i \in \mathcal{I}$, is $90 s, 60 s$, and $70 s$, respectively. See [10] for further comments on the above quantities.

The objective of this work is to derive a plan for optimal usage of the three fuels described above during 24 hours of operation. Optimal usage is defined as maximizing the profit in terms of two business objectives: efficiency and controllability. The models used in this work for business objectives are based on the following: Efficiency deals with a measure of efficiency ${ }^{2}$ of the

\footnotetext{
${ }^{2}$ Remark that the term efficiency in this work often is referred to as production in power plant community.
}

conversion of a fuel into electricity and controllability is a measure of the plant's capability to change the production level. In addition to maximizing the profit, the production level of the plant is to follow a time varying reference $y_{r}(t)$ (also called a production plan) as closely as possible.

The efficiency objective is modeled as

$$
y_{e}(\boldsymbol{z})=\boldsymbol{\gamma}^{T} \tilde{\boldsymbol{Q}} \boldsymbol{z}+\boldsymbol{\gamma}^{T} \boldsymbol{b},
$$

where

$$
\begin{aligned}
\tilde{\boldsymbol{Q}} & =\operatorname{diag}\left(\boldsymbol{e}_{x}\right) \boldsymbol{C}, \quad \boldsymbol{e}_{x}=(10.77,18.87,15.77), \\
\boldsymbol{b} & =(-1.76,1.85,-0.37), \boldsymbol{\gamma}=(1,1,1),
\end{aligned}
$$

with $\boldsymbol{C}$ as in (1). The value of the entries of $\boldsymbol{e}_{x}$ and $\boldsymbol{b}$ has been established using measurement data provided by DONG Energy ${ }^{3}$. The elements of $\boldsymbol{e}_{x}$ are conversion factors from mass flows to electrical-energy flows, and the entries of $\boldsymbol{b}$ are energy used or generated in preprocessing of the fuels.

The controllability objective is modeled as

$$
y_{c}(\boldsymbol{z}, t)=\boldsymbol{\vartheta}(t) \boldsymbol{z}+\zeta(t),
$$

where

$$
\begin{gathered}
\boldsymbol{\vartheta}(t)= \begin{cases}0 & y_{r}(t) \in S_{1}=\{s \in \mathbb{R} \mid 0 \leq s \leq 200\} \\
\frac{\boldsymbol{\xi}^{T} \tilde{\boldsymbol{Q}}}{y_{r}(t)} & y_{r}(t) \in S_{2}=\{s \in \mathbb{R} \mid 200<s<360\} \\
0 & y_{r}(t) \in S_{3}=\{s \in \mathbb{R} \mid 360 \leq s \leq 400\},\end{cases} \\
\zeta(t)= \begin{cases}0.133 & y_{r}(t) \in S_{1} \\
\frac{\boldsymbol{\xi}^{T} \boldsymbol{b}}{y_{r}(t)} & y_{r}(t) \in S_{2} \\
0.133 & y_{r}(t) \in S_{3},\end{cases}
\end{gathered}
$$

with $\boldsymbol{\xi}=(0.267,0.534,0.534)$ and $S_{1}, S_{2}$, and $S_{3}$ denote different operating regions. The operating regions arise as maximum temperature gradients are imposed in the boiler due to wear and tear of the building materials. Therefore, the controllability measure also changes depending on the current production.

The value of the objectives have been established using price data available at Nord Pool and in collaboration with DONG Energy by using their heuristics. That is, current and historic prices of electricity, which is available online has been used as price of the efficiency measure. The instantaneous profit flow is formulated as in [10], i.e.,

$$
g_{p}(\boldsymbol{z}, t)=\boldsymbol{\Theta}(t) \boldsymbol{z}+\tilde{\varphi}(t)
$$

where

$$
\begin{aligned}
\boldsymbol{\Theta}(t) & =p_{R 1}(t) \boldsymbol{\gamma}^{T} \tilde{\boldsymbol{Q}}-\boldsymbol{p}_{C}^{T} \boldsymbol{C}+p_{R 2}(t) \boldsymbol{\vartheta}(t), \\
\tilde{\varphi}(t) & =p_{R 1}(t) \boldsymbol{\gamma}^{T} \boldsymbol{b}+p_{R 2}(t) \zeta(t),
\end{aligned}
$$

with $\boldsymbol{p}_{C}$ the price of the different fuels, and $p_{R 1}$ and $p_{R 2}$ are prices imposed on the two business objectives, efficiency and controllability respectively. The functions in (4) are in this work assumed sufficiently smooth $\left(C^{5}\right.$ is enough as shown in Appendix I). Further description

${ }^{3}$ DONG Energy is a power producer in Denmark. 
and explanation of the above quantities can be found in [14], [12], [13], and [10].

A prognosis of the next days's electricity consumption is established by Energinet. $\mathrm{dk}^{4}$, which is responsible for the electrical grid in Denmark. The estimated electricity consumption in an area (e.g. West Denmark) is divided between the different electricity producers in accordance with the bids on Nord Pool; and thus, a production plan is generated for each producer. The production plan used in this work is an approximation of a production plan delivered by DONG Energy. These are depicted in Figure 2 (for more details on the production plan see [13]). The plant should follow the generated production plan

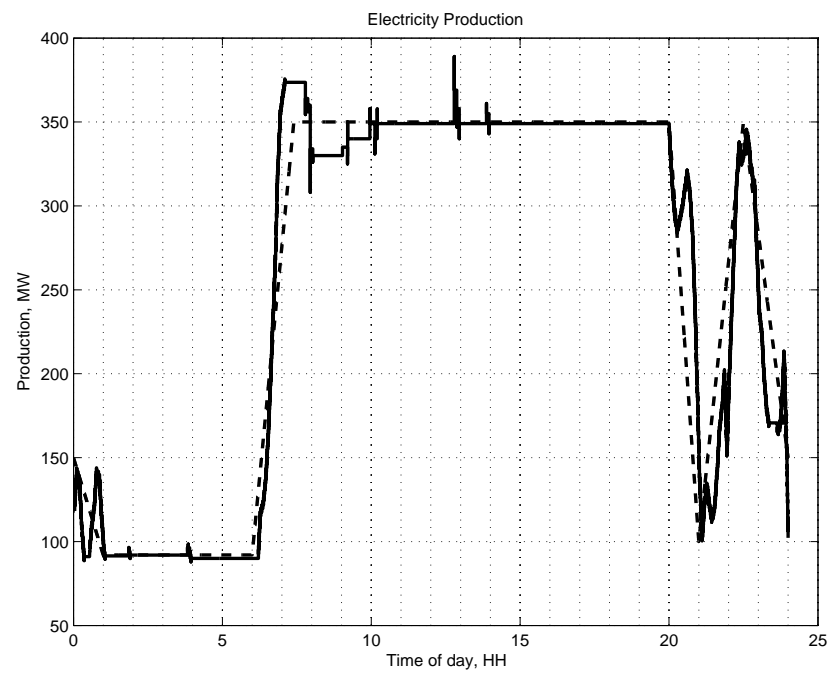

Fig. 2. Production plan for June 29th, 2008. The data used to generate this plot has been delivered by DONG Energy (solid). The approximation used in this work consists of piece-wise affine function (dashed).

such that power balance can be upheld. The tracking requirement is included by adding the quadratic tracking error,

$$
\begin{aligned}
t_{e}(\boldsymbol{z}, t)= & \left\|y_{e}(\boldsymbol{z})-y_{r}(t)\right\|^{2} \\
= & y_{e}(\boldsymbol{z})^{2}-2 y_{r}(t) y_{e}(\boldsymbol{z})+y_{r}(t)^{2} \\
= & \boldsymbol{z}^{T} \tilde{\boldsymbol{Q}}^{T} \boldsymbol{\gamma} \boldsymbol{\gamma}^{T} \tilde{\boldsymbol{Q}} \boldsymbol{z}+2 \boldsymbol{b}^{T} \boldsymbol{\gamma} \boldsymbol{\gamma}^{T} \tilde{\boldsymbol{Q}} \boldsymbol{z}+\boldsymbol{b}^{T} \boldsymbol{\gamma} \boldsymbol{\gamma}^{T} \boldsymbol{b} \\
& -2 y_{r}(t) \boldsymbol{\gamma}^{T} \tilde{\boldsymbol{Q}} \boldsymbol{z}+y_{r}(t)^{2}
\end{aligned}
$$

as a penalty term in the objective function in the optimization. This approach has been taken as the computational complexity is lowered by including the requirement in the objective function and not as an additional constraint [11].

As a result, the optimization problem is expressed as

$$
\begin{array}{ll}
\max _{\boldsymbol{u} \in \mathcal{U}} & \int_{0}^{T} f(\boldsymbol{z}, t) d t \\
\text { subject to } & \dot{\boldsymbol{z}}(t)=\boldsymbol{A} \boldsymbol{z}(t)+\boldsymbol{B} \boldsymbol{u}(t),
\end{array}
$$

where the input space is given by

$$
\mathcal{U}=\left\{\boldsymbol{u} \in \mathbb{R}_{+}^{3} \mid \boldsymbol{e}_{u}^{T} \boldsymbol{u} \leq c_{u}, \boldsymbol{e}_{u}>0\right\},
$$

${ }^{4}$ Energinet.dk a Danish Transmission System Operator, TSO. with $\boldsymbol{e}_{u}=\boldsymbol{e}_{x}$ and $c_{u}=400-\gamma^{T} \boldsymbol{b}$ as in [10], and $f$ given by

$$
\begin{aligned}
f(\boldsymbol{z}, t) & =g_{p}(\boldsymbol{z}, t)-\beta_{q} t_{e}(\boldsymbol{z}, t) \\
& =-\boldsymbol{z}^{T} \boldsymbol{Q} \boldsymbol{z}+2 \boldsymbol{q}(t)^{T} \boldsymbol{z}+\varphi(t),
\end{aligned}
$$

with

$$
\begin{aligned}
\boldsymbol{Q} & =\beta_{q} \tilde{\boldsymbol{Q}}^{T} \boldsymbol{\gamma} \boldsymbol{\gamma}^{T} \tilde{\boldsymbol{Q}} \\
\boldsymbol{q}(t)^{T} & =\frac{1}{2} \boldsymbol{\Theta}(t)+\beta_{q}\left(y_{r}(t) \boldsymbol{\gamma}^{T} \tilde{\boldsymbol{Q}}-\boldsymbol{b}^{T} \boldsymbol{\gamma} \boldsymbol{\gamma}^{T} \tilde{\boldsymbol{Q}}\right) \\
\varphi(t) & =\tilde{\varphi}(t)+\beta_{q}\left(\boldsymbol{b}^{T} \boldsymbol{\gamma} \boldsymbol{\gamma}^{T} \boldsymbol{b}-y_{r}(t)^{2}\right),
\end{aligned}
$$

and $\beta_{q}$ a positive weighting factor, which can be described as a reference penalty factor.

In the remaining sections, this paper deals with solving the optimization problem in (6).

\section{Continuous Optimization}

In this section, Pontryagin's maximum principle will be applied to the optimization problem described above.

However, first, notice that by replacing the (noncontinuous) functions $\boldsymbol{q}$ and $\varphi$ by continuous approximations, such that $f$ becomes continuous, Filippov's existence theorem [5, pp. 199] may be applied. Hence, in the continuous case, there exists an optimal solution to the optimization problem. Nonetheless, the question of existence for the discontinuous case will not be pursued here (see e.g. [5, p. 386] for a statement in this direction) since numerical solutions to the optimization problem are to be used, and since the above continuous approximations may be chosen with any given precision [23].

The Hamiltonian approach is now used, i.e., necessary conditions are deduced, by means of Pontryagin's maximum principle, to obtain candidates for optimal solutions.

The Hamiltonian for the optimization problem is

$$
\begin{aligned}
& H(\boldsymbol{z}, \boldsymbol{u}, \boldsymbol{\lambda}, t) \\
& \quad=f(\boldsymbol{z}, t)+\boldsymbol{\lambda}^{T}(\boldsymbol{A} \boldsymbol{z}+\boldsymbol{B} \boldsymbol{u}),
\end{aligned}
$$

and thus, the adjoint equation is given by

$$
\begin{aligned}
\dot{\boldsymbol{\lambda}}(t) & =-\frac{\partial H(\boldsymbol{z}(t), \boldsymbol{u}(t), \boldsymbol{\lambda}(t), t)}{\partial \boldsymbol{z}} \\
& =-\frac{\partial f(\boldsymbol{z}(t), t)}{\partial \boldsymbol{z}}-\boldsymbol{A}^{T} \boldsymbol{\lambda}(t) \\
& =2 \boldsymbol{Q} \boldsymbol{z}(t)-2 \boldsymbol{q}(t)-\boldsymbol{A}^{T} \boldsymbol{\lambda}(t),
\end{aligned}
$$

with the transversality condition $\boldsymbol{\lambda}(T)=\mathbf{0}$. Point-wise maximization of $H$ then yields

$$
\begin{aligned}
\max _{\boldsymbol{u} \in \mathcal{U}} H(\boldsymbol{z}(t), \boldsymbol{u}, \boldsymbol{\lambda}(t), t)= & f(\boldsymbol{z}(t), t)+\boldsymbol{\lambda}(t)^{T} \boldsymbol{A} \boldsymbol{z}(t) \\
& +\max _{\boldsymbol{u} \in \mathcal{U}} \underbrace{\boldsymbol{\lambda}(t)^{T} \boldsymbol{B}}_{\boldsymbol{\sigma}(t)^{T}} \boldsymbol{u} .
\end{aligned}
$$

Note that $\boldsymbol{\sigma}(t)$ is not known. However, by examining the sign of the coordinates of the vector $\boldsymbol{\sigma}(t)$, it is possible to 
determine the following properties of the optimal input, $\boldsymbol{u}^{*}(t)$

$$
\sigma_{i}(t)<0 \Rightarrow u_{i}^{*}(t)=0
$$

Now, let

$$
\mathcal{U}(t)=\left\{\boldsymbol{u} \in \mathcal{U} \mid u_{i}=0 \text { if } \sigma_{i}(t)<0\right\},
$$

and let $\boldsymbol{E}(t)$ be the matrix which by projection removes the negative elements of $\boldsymbol{\sigma}(t)$, e.g., if $\sigma_{1}(t)<0$ and $\sigma_{2}(t), \sigma_{3}(t) \geq 0$, we have

$$
\boldsymbol{E}(t)=\left[\begin{array}{lll}
0 & 1 & 0 \\
0 & 0 & 1
\end{array}\right]
$$

Note that the mapping $\boldsymbol{E}(t)$ is injective when restricted to $\mathcal{U}(t)$.

Let $\tilde{\boldsymbol{\sigma}}(t)=\boldsymbol{E}(t) \boldsymbol{\sigma}(t)$. Now, two cases remain to be analyzed.

$$
\begin{array}{ll}
\text { Case 1: } & \sum \tilde{\sigma}_{i} \neq 0 . \\
\text { Case 2: } & \tilde{\boldsymbol{\sigma}}(t)=\mathbf{0} .
\end{array}
$$

In Case 1 , the optimal control input $\boldsymbol{u}^{*}(t)$ is found from

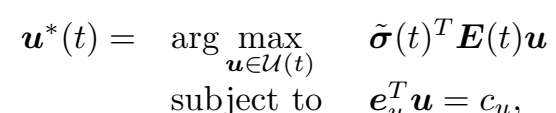

which, for each time $t$, searches through corners of a 2simplex, 1-simplex, or 0-simplex in $\mathbb{R}^{3}$.

Case 2 is a singular optimal control problem [2], [4]. The optimal control input in Case 2 is found from (27) in Appendix I as

$$
\boldsymbol{C}_{u}(t) \boldsymbol{u}^{*}(t)=\boldsymbol{C}_{z}(t) \boldsymbol{z}(t)+\boldsymbol{C}_{\tau}(t), \quad \boldsymbol{u}^{*}(t) \in \mathcal{U}(t) .
$$

Here, the time dependence which was left out in the appendix is reintroduced as the entire time horizon is considered.

Now by introducing the following relation

$$
\boldsymbol{u}^{*}(t)=\boldsymbol{E}(t)^{T} \tilde{\boldsymbol{u}}^{*}(t),
$$

and inserting it in (11), the following equation is obtained.

$$
\boldsymbol{C}_{u}(t) \boldsymbol{E}(t)^{T} \tilde{\boldsymbol{u}}^{*}(t)=\boldsymbol{C}_{z}(t) \boldsymbol{z}(t)+\boldsymbol{C}_{\tau}(t) .
$$

Since $\boldsymbol{C}_{u}(t) \boldsymbol{E}(t)^{T}$ is square and non-singular, $\tilde{\boldsymbol{u}}^{*}(t)$ is given by

$$
\begin{aligned}
\tilde{\boldsymbol{u}}^{*}(t)= & \left(\boldsymbol{C}_{u}(t) \boldsymbol{E}(t)^{T}\right)^{-1} \boldsymbol{C}_{z}(t) \boldsymbol{z}(t) \\
& +\left(\boldsymbol{C}_{u}(t) \boldsymbol{E}(t)^{T}\right)^{-1} \boldsymbol{C}_{\tau}(t) .
\end{aligned}
$$

Now, a $\boldsymbol{u}^{*}(t) \in \mathcal{U}(t)$ can again be computed by using (12),

$$
\begin{aligned}
\boldsymbol{u}^{*}(t)= & \boldsymbol{E}(t)^{T}\left(\boldsymbol{C}_{u}(t) \boldsymbol{E}(t)^{T}\right)^{-1} \boldsymbol{C}_{z}(t) \boldsymbol{z}(t) \\
& +\boldsymbol{E}(t)^{T}\left(\boldsymbol{C}_{u}(t) \boldsymbol{E}(t)^{T}\right)^{-1} \boldsymbol{C}_{\tau}(t) .
\end{aligned}
$$

In conclusion, in Case 1, the optimal control input is an open loop controller; and in Case 2, a combination of a state feedback and a time varying feedforward. This combination will in the following be denoted "feedback/feedforward".
Now, a strategy for finding the optimal control input is devised; however, the switching function $\boldsymbol{\sigma}(t)$, which is required in the control law, is unknown and thus the time instances of switching between the two cases (and different $\boldsymbol{E}(t))$ can not be determined.

The next two sections of this paper will present a solution to this problem. It will be based on an approximated solution to the optimization problem. The approximated solution will subsequently be used to solve the adjoint equation and thus obtain an approximated solution for $\boldsymbol{\sigma}(t)$. The approximated solution will be found using a discrete time formulation of the optimization problem. This procedure is explained in the next section.

\section{Discrete Optimization}

The optimization problem in (6) has been addressed in [10] where the tracking of the reference was formulated as a constraint. As a consequence, it has not been modeled in the objective function. This is done in this work. In this section, we will apply the procedure in [10] to the quadratic objective function, i.e., we will formulate the optimization problem in discrete time. In Appendix II, lifting [6] is applied to the objective function in (6) to obtain a discrete-time expression. However, some assumptions are imposed. In particular, it is assumed that $\boldsymbol{q}(t)$ and $\varphi(t)$ can be approximated by piecewise constant functions, and that the control $\boldsymbol{u}$ is piecewise constant.

The discrete time objective function can be formulated as

$$
\sum_{k=0}^{N-1}\left(\left[\begin{array}{ll}
\boldsymbol{z}_{k}^{T} & \boldsymbol{u}_{k}^{T}
\end{array}\right] \boldsymbol{N}\left[\begin{array}{l}
\boldsymbol{z}_{k} \\
\boldsymbol{u}_{k}
\end{array}\right]+\boldsymbol{M}_{z_{k}} \boldsymbol{z}_{k}+\boldsymbol{M}_{u_{k}} \boldsymbol{u}_{k}+h \varphi_{k}\right),
$$

where

$$
\boldsymbol{N}=-\left[\begin{array}{ll}
\boldsymbol{N}_{z z} & \boldsymbol{N}_{z u} \\
\boldsymbol{N}_{u z} & \boldsymbol{N}_{u u}
\end{array}\right]
$$

with the matrices $\boldsymbol{N}_{z z}, \boldsymbol{N}_{z u}, \boldsymbol{N}_{u z}, \boldsymbol{N}_{u u}, \boldsymbol{M}_{z_{k}}$, and $\boldsymbol{M}_{u_{k}}$ as given by (32) and (38) in Appendix II. The optimization problem in (6) can be rewritten by introducing the following notation

$$
\begin{aligned}
\tilde{\boldsymbol{\Phi}}=\left[\begin{array}{c}
\boldsymbol{I} \\
\boldsymbol{\Phi} \\
\boldsymbol{\Phi}^{2} \\
\vdots \\
\boldsymbol{\Phi}^{N-2}
\end{array}\right], \quad \tilde{\boldsymbol{\varphi}}=\left[\begin{array}{c}
\varphi_{0} \\
\varphi_{1} \\
\varphi_{2} \\
\vdots \\
\varphi_{N-1}
\end{array}\right], \quad \mathbf{1}=\left[\begin{array}{c}
1 \\
1 \\
1 \\
\vdots \\
1
\end{array}\right], \\
\tilde{\boldsymbol{\Gamma}}=\left[\begin{array}{cccc}
\boldsymbol{0} & \mathbf{0} & \cdots & \mathbf{0} \\
\boldsymbol{\Gamma} \boldsymbol{\Gamma} & \mathbf{0} & \ddots & \mathbf{0} \\
\vdots & \vdots & \ddots & \mathbf{0} \\
\boldsymbol{\Phi}^{N-3} \boldsymbol{\Gamma} & \boldsymbol{\Phi}^{N-4} \boldsymbol{\Gamma} & \cdots & \boldsymbol{\Gamma}
\end{array}\right]
\end{aligned}
$$

and the matrices $\boldsymbol{\Phi}=e^{\boldsymbol{A} h}$ and $\boldsymbol{\Gamma}=\int_{0}^{h} e^{\boldsymbol{A} s} \boldsymbol{B} d s$ are the discrete time equivalences of the system matrices given in (1). Furthermore, $\varphi_{k}$ is given by (29) in Appendix II.

Using the above discrete time system and considering $\boldsymbol{z}_{k}$ as a function of $\boldsymbol{z}_{0}$ and $\boldsymbol{u}_{k}$, it is possible to formulate 
the following optimization problem, which is the discrete time equivalent of $(6)$

$$
\max _{\boldsymbol{v} \in \mathcal{V}} \boldsymbol{v}^{T} \boldsymbol{W} \boldsymbol{v}+\boldsymbol{L} \boldsymbol{v}+g
$$

where

$$
\begin{aligned}
\boldsymbol{v}= & {\left[\begin{array}{c}
\boldsymbol{u}_{0} \\
\boldsymbol{u}_{1} \\
\vdots \\
\boldsymbol{u}_{N-2}
\end{array}\right], } \\
\mathcal{V}= & \left\{\boldsymbol{v} \in \mathbb{R}^{m(N-1)} \mid \boldsymbol{v}_{i} \in \mathcal{U}, i \in\{0,1,2, \ldots, N-2\}\right\}, \\
\boldsymbol{W}= & -\tilde{\boldsymbol{\Gamma}}^{T}\left(\boldsymbol{I} \otimes \boldsymbol{N}_{z z}\right) \tilde{\boldsymbol{\Gamma}}-\tilde{\boldsymbol{\Gamma}}^{T}\left(\boldsymbol{I} \otimes \boldsymbol{N}_{z u}\right) \\
& -\left(\boldsymbol{I} \otimes \boldsymbol{N}_{u z}\right) \tilde{\boldsymbol{\Gamma}}-\boldsymbol{I} \otimes \boldsymbol{N}_{u u}, \\
\boldsymbol{L}= & \mathbf{1}^{T} \otimes \boldsymbol{M}_{u_{k}}+\left(\mathbf{1}^{T} \otimes \boldsymbol{M}_{z_{k}}\right) \tilde{\boldsymbol{\Gamma}} \\
& -\boldsymbol{z}_{0}^{T} \tilde{\boldsymbol{\Phi}}^{T}\left(\boldsymbol{I} \otimes \boldsymbol{N}_{z u}+\boldsymbol{I} \otimes \boldsymbol{N}_{u z}^{T}\right) \\
& -\boldsymbol{z}_{0}^{T} \tilde{\boldsymbol{\Phi}}^{T}\left(\boldsymbol{I} \otimes \boldsymbol{N}_{z z}+\boldsymbol{I} \otimes \boldsymbol{N}_{z z}^{T}\right) \tilde{\boldsymbol{\Gamma}}, \\
g= & \left(\mathbf{1}^{T} \otimes \boldsymbol{M}_{z_{k}}\right) \tilde{\boldsymbol{\Phi}} \boldsymbol{z}_{0}+h \mathbf{1}^{T} \tilde{\boldsymbol{\varphi}}-\boldsymbol{z}_{0}^{T} \tilde{\boldsymbol{\Phi}}^{T}\left(\boldsymbol{I} \otimes \boldsymbol{N}_{z z}\right) \tilde{\boldsymbol{\Phi}} \boldsymbol{z}_{0},
\end{aligned}
$$

with $\otimes$ the Kronecker product.

The optimization in (14) has been implemented using YALMIP with the following constants used for the parameters in (32) and (38)

$$
h=192 s, \quad \beta_{q}=0.05, \quad T=86400 s, \quad N=450,
$$

and solved using the quadratic solver BPMPD. Remark that the sampling time of $192 s$ yielding 450 discrete points in time is close to the limit of the capabilities of the computer used for the optimization.
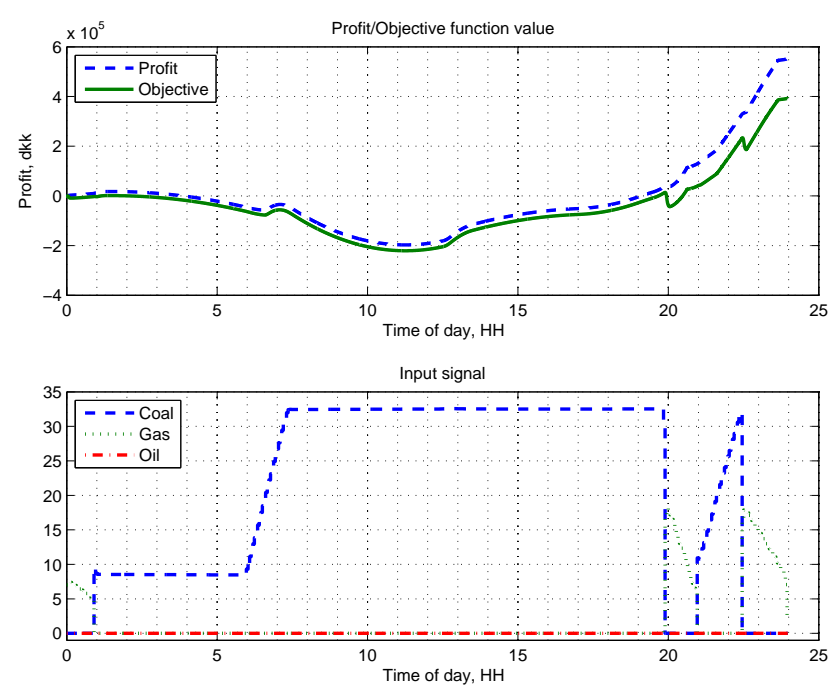

Fig. 3. The result of the discrete optimization. Top: The profit and value of objective function during 24 hours. Bottom: The control signal for the different fuel systems.

Figure 3-top depicts the profit and the value of the objective function. Remark that the word "profit" is used for the real profit of the company, i.e., the objective function value without the quadratic tracking penalty, $t_{e}$, and the expression "value of the objective function" is used when the quadratic tracking penalty is included. The profit computed in this work is in the same order of magnitude as the results obtained in [10] where the tracking requirement was implemented as a constraint instead of included in the objective function as in this work.

The usage of fuels are also comparable to [10] except that around 7:00 when the gas system is not used, as seen in Figure 3-bottom.

\section{Optimal Feedback}

In this section, the idea of a continuous feedback/feedforward from Section III is revisited. The reason for this is the usual robust behavior of a feedback system with regards to noise compared with pure feedforward control (this is further discussed in Section VI).

Let us begin by remarking that an essential part of the optimization problem is to follow the predefined reference. In particular, there should be no market situation such that a large deviation from reference is beneficial. This, of course, depends on the value of $\beta$ which in this work has been chosen such that the above is satisfied.

As stated at the end of Section III, the reason for not using the feedback/feedforward solution is that $\boldsymbol{\sigma}(t)$ is unknown. To compensate for this, we introduce an algorithm whose purpose is to approximate $\boldsymbol{\sigma}(t)$. In short, we need to approximate $\boldsymbol{\lambda}(t)$ which depends on $\boldsymbol{z}(t)$. As an input for the algorithm we use the discretized solution $\boldsymbol{z}(t)=\left(\boldsymbol{z}_{0}, \ldots, \boldsymbol{z}_{N-2}\right)$ given by $\tilde{\boldsymbol{\Phi}} \boldsymbol{z}_{o}+\tilde{\boldsymbol{\Gamma}} \boldsymbol{v}$ as described in the previous section. Note that $\boldsymbol{z}(t)$ can be viewed as a small perturbation of the optimal solution $z^{*}(t)$. As a result, the algorithm is, as follows:

1. Use $\boldsymbol{z}(t)$ to obtain $\boldsymbol{\lambda}(t)$ in (7) with transversality condition $\boldsymbol{\lambda}(T)=\mathbf{0}$. In simulations, this step is preformed using Matlab's ode 45 .

2 . Use $\boldsymbol{\lambda}(t)$ to compute $\boldsymbol{\sigma}(t)=\boldsymbol{B}^{T} \boldsymbol{\lambda}(t)$ and the projection $\boldsymbol{E}(t)$ as described in Section III.

3. For each time $t$ determine, by evaluating $\boldsymbol{\sigma}(t)$, whether case 1 or 2 in (9) holds:

I. If case 1 use (10) to compute $\boldsymbol{u}^{*}(t)$.

II. If case 2 use $\boldsymbol{\lambda}(t)$ and $\boldsymbol{E}(t)$ to compute $\boldsymbol{C}_{i}(t), i=z, u, t$ given by (28). Then compute $\boldsymbol{u}^{*}(t)$ using (13). We remark that due to numerical imprecision, in simulations, we have placed a band around 0 of width 1 in which all elements are set to zero.

4. Use $\boldsymbol{u}^{*}(t)$ to obtain $\boldsymbol{z}(t)$ in (1). In simulations, this step is preformed using Matlab's ode45.

5. Return to 1.

The switching function, $\boldsymbol{\sigma}(t)=\boldsymbol{B}^{T} \boldsymbol{\lambda}(t)$ is depicted in Figure 4, where the solution to the adjoint equation $\boldsymbol{\lambda}(t)$ is computed using the discrete state trajectory $\left(\boldsymbol{z}\left(t_{k}\right)\right), t_{k} \in\{0, h, 2 h, \cdots,(N-1) h\}$, i.e., the first iteration of the algorithm described above. After 300 iterations, the above algorithm shows no sign of convergence. This is illustrated in Figure 5, where the graph of $\boldsymbol{\sigma}(t)$ is depicted for iteration 295-300. The 


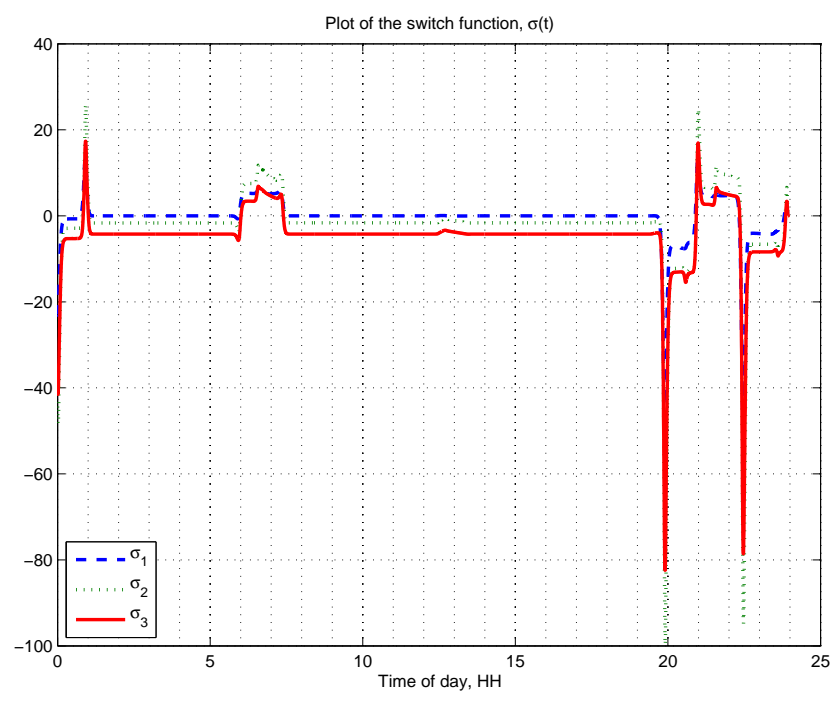

Fig. 4. Graph of the switching function obtained by using the state trajectory from the discrete optimization.

solutions switch between two different profiles, this can be explained heuristically as follows: First, note that the adjoint equation is solved backwards and hence an equivalent problem can be stated as

$$
\dot{\Lambda}=\boldsymbol{A}^{T} \boldsymbol{\Lambda}+\boldsymbol{c},
$$

with

$$
\begin{aligned}
\boldsymbol{c}= & -2 \boldsymbol{Q} \boldsymbol{z}(t)+2 \boldsymbol{q}(t) \\
= & -2 \beta_{q} \tilde{\boldsymbol{Q}}^{T} \boldsymbol{\gamma} \boldsymbol{\gamma}^{T} \tilde{\boldsymbol{Q}}+2 \beta_{q}\left(\tilde{\boldsymbol{Q}}^{T} \boldsymbol{\gamma} y_{r}(t)-\tilde{\boldsymbol{Q}}^{T} \boldsymbol{\gamma} \boldsymbol{\gamma}^{T} \boldsymbol{b}\right) \\
& +\boldsymbol{\Theta}^{T}(t) \\
= & -2 \beta_{q} \tilde{\boldsymbol{Q}}^{T} \boldsymbol{\gamma}\left(y_{e}(\boldsymbol{z}(t))-y_{r}(t)\right)+\boldsymbol{\Theta}^{T}(t) .
\end{aligned}
$$

Now, consider $\boldsymbol{c}$ as an input to system (15). Since $\boldsymbol{A}^{T}$ is stable $\boldsymbol{\Lambda}(t)$ will track $\boldsymbol{c}$ and hence if $\boldsymbol{c}$ oscillates then $\boldsymbol{\Lambda}(t)$ will oscillates. In particular, if the sign of $c_{i}$ changes so does the sign of $\Lambda_{i}(t)$.

Now, let $i$ be either 3,6 , or 9 and assume, in iteration $\mathrm{k}$, that $c_{i}>0$ on an interval, $T_{1}$. This will result in $\Lambda_{i}(t)>0$ for $t \in T_{2} \subset T_{1}$. Then in iteration $\mathrm{k}+1, \sigma_{i / 3}(t)>0, t \in$ $T_{2}$, and according to (10) this will result in maximum efficiency and $c_{i}$ will according to (16) be negative. This will, in return, yield a negative $\sigma_{i / 3}(t), t \in T_{3} \subset T_{2}$; and thus, the proposed algorithm will not converges.

Moreover, the oscillating behavior of the algorithm described above is indicated by Figure 4. More precisely, the initial estimate of $\boldsymbol{\sigma}(t)$ is less than zero in [0,0:15]. According to $(8), \boldsymbol{u}(t)=\mathbf{0}, t \in[0,0: 15]$. As a result, the efficiency output $y_{e}$ deviates from the reference $y_{r}$ causing the increase of the error calculated by (5). This behavior is also present in the intervals [19:45,21] and $[22: 30,24]$ and in the intervals $[0: 45,1: 05][6,7: 30]$ and [21, 22:30]. Due to the choice of $\beta_{q}$, as discussed above, this behavior cannot result in an optimal solution. Therefore, subsequent iterations will not improve the estimate of the parameters, and in particular not $\boldsymbol{\sigma}(t)$ as seen in
Figure 5. Remark that it is well known that numerical problems may appear in problems with singular arcs. The authors are not aware of any general methods (numerical or theoretical) which prove convergence to co-states associated to optimal solutions, see however [9]. In conclusion the above method has to be modified in order to obtain reference tracking as discussed above.
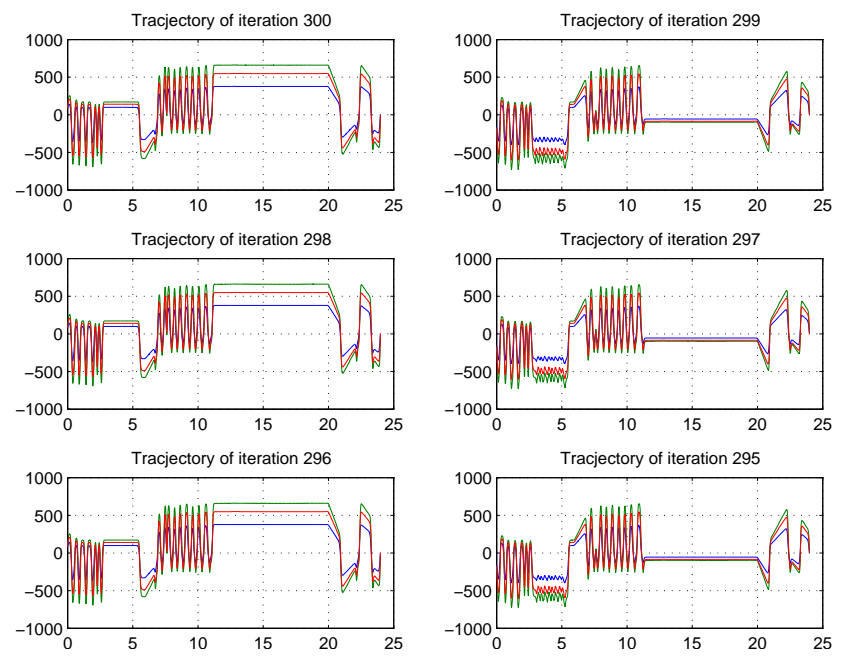

Fig. 5. Graph of the switching function calculated in iterations 295-300.

Therefore, some heuristics are introduced to avoid the reference deviations (and non-optimal behavior). From the discrete optimization, it is observed that only one fuel is used at the time, which has also been suggested by earlier work [10], [13]. As a consequence, $\sigma_{i, j}(t)<0$ at any given time, where the notation $x_{i, j}$ means coordinates $i$ and $j$ of the vector $x$. Therefore, the introduced heuristics is to use only the one element of $\boldsymbol{u}(t)$ corresponding to the largest element of $\boldsymbol{\sigma}(t)$. To recapitulate, item 3. in the above algorithm is replaced by

3'. Determine the largest element of $\sigma(t)$, say $\sigma_{l}(t)$, and let $E(t)$ be the $1 \times 3$ matrix with 1 at place $l$ and zero's elsewhere, and then, use (13) to compute $u^{*}(t)$.

Using this concept, it is possible to obtain an control input as described in Section III, which yields better behavior. When applied, this input strategy will provide another state trajectory and thus a different trajectory of $\boldsymbol{\sigma}(t)$. For the given case study, this procedure stabilizes ${ }^{5}$, which might not be true in general. Remark that in order to conduct a rigorous mathematical discussion about the convergence properties, a mathematical model of the above algorithm has to be devised. Such a model can only be an approximation of the above algorithm due to the introduced heuristics when defining $\boldsymbol{E}(t)$. This is further complicated by the discrete behavior of $\boldsymbol{\sigma}(t)$ and $\boldsymbol{E}(t)$. However, by the heuristic construction of $\boldsymbol{E}(t)$, reference

\footnotetext{
${ }^{5}$ The procedure has been executed 300 times and after step four it stabilizes.
} 
tracking is maintained. This implies that (16) becomes

$$
\boldsymbol{c}=-2 \beta_{q} \tilde{\boldsymbol{Q}}^{T} \gamma \epsilon+\boldsymbol{\Theta}^{T}(t)
$$

for small $\epsilon$, i.e., $\boldsymbol{c}$ is not affected by the iterations due to reference tracking. As a result, only a small perturbation of the stable linear system (15) is introduced in each iteration. This results in convergence of the proposed algorithm.

Note that it is possible to solve (15) using (17) with $\epsilon=0$ without iterating the proposed algorithm.

In this paper, the first four iterations of this procedure has been applied, where the state trajectory from the discrete optimization is used for the initial iteration. Figure 6 depicts the convergence of the switching function from the different iterations, where $\sigma_{11}$ denotes coordinate one for iteration one, $\sigma_{12}$ coordinate one from iteration two, etc. As seen in the figure, Coordinate three
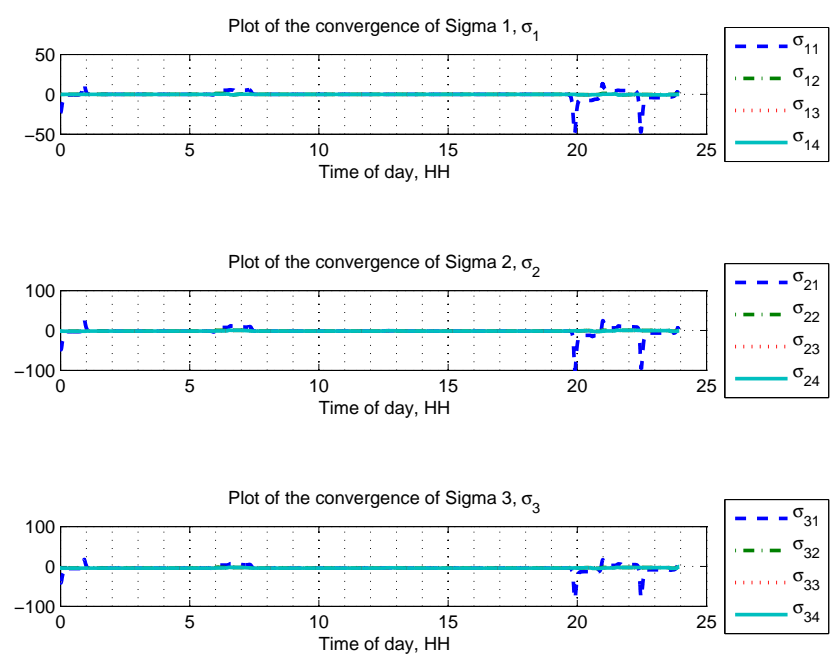

Fig. 6. Convergence of the coordinates of the switching function - four iterations are depicted. Note that iteration two, three, and four are almost on top of each other.

always converges to a value less than zero. Thus, the oil system should not be used at all and could be omitted when the plant is instrumented. Coordinate two is less than zero for large periods during the day; hence, gas should not be used during these periods. Coordinate one, on the other hand, is zero most of the time.

The input strategy for each of the four iterations has been applied to the model of the plant. Figure 7 depicts the graphs of the resulting objective-function values for the four iterations of the adjoint equation along with the objective function value from the discrete optimizations. The legends refer to the value of the discrete objective function and continuous objective function from iteration $1,2,3$, and 4 . As seen in the figure, the value of the objective function increases substantially from the discrete to the first iteration and from the first iteration to the second iteration. However, iteration three and four do not change the value of the objective function significantly.

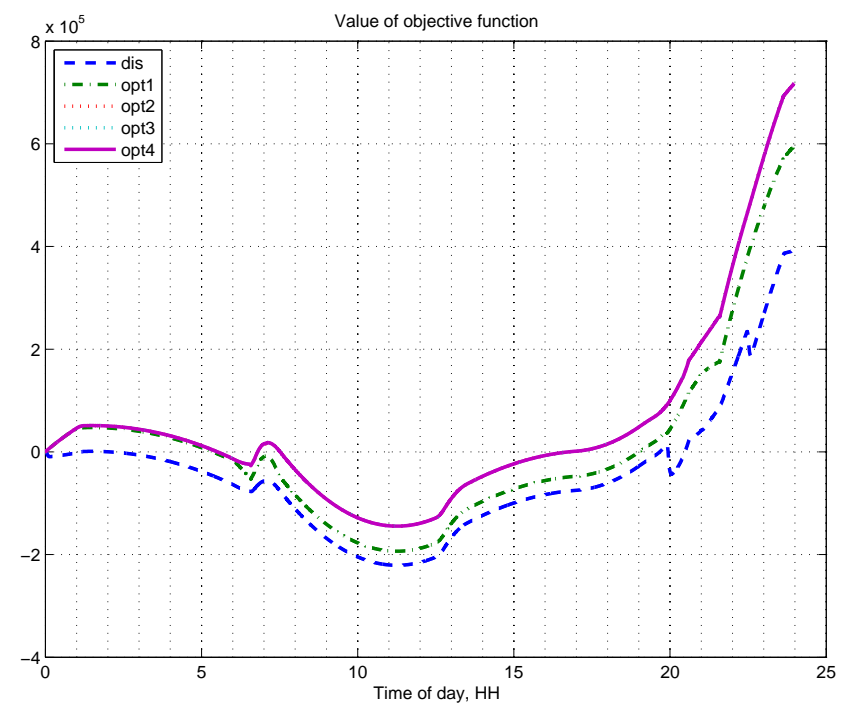

Fig. 7. The value of the objective function when using the input from the discrete optimization and the four iterations of the switching function. Note that the graph of iteration two, three, and four are almost on top of each other.

The final input strategy is evaluated in Figure 8, where the top figure depicts the profit and objective function value, and the bottom figure depicts optimal input. As seen in the top figure, the profit and objective
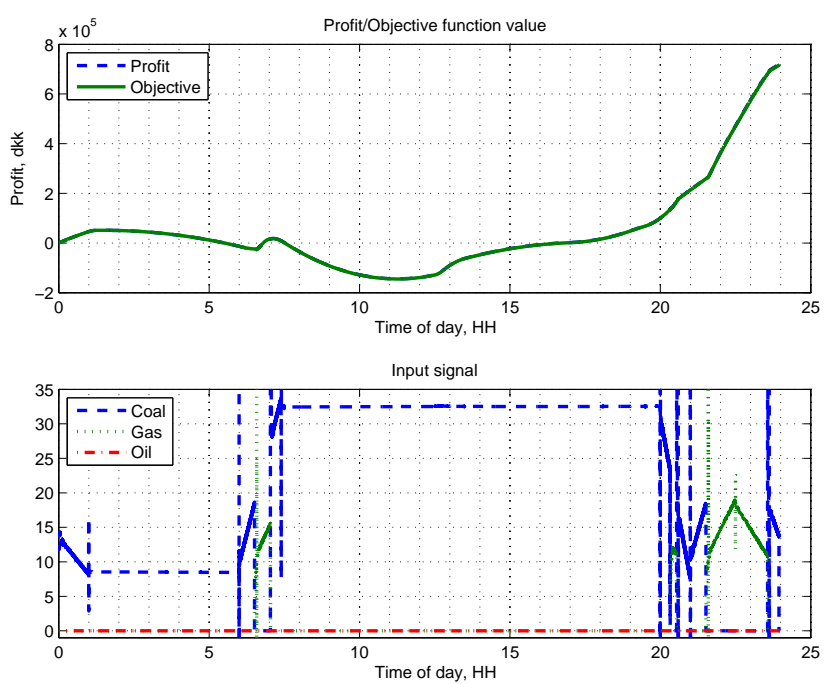

Fig. 8. Result of using the optimal input from the fourth iteration. Top: The profit and value of objective function during 24 hours. Bottom: The control signal for the different fuel systems. The spikes in the control signal arise from the switching between the sets $S_{1}$, $S_{2}$, and $S_{3}$.

function values are very close to each other. Thus, the production tracks the reference closely when the optimal feedback/feedforward is used. The final profit is approximately $168000[D K K]$ or $30 \%$ larger than what was obtained using the input from the discrete optimization. Furthermore, the optimal input is different from the input obtained in the discrete optimization. This is specially seen in the usage of gas in the period 6:30-7:00 as 
depicted in bottom figure. The spikes in the input signal are due to the discontinuous switches in (3). However, these spikes do not affect the output as they are of very short time span. Furthermore, notice that gas is used briefly in the period 20:20-20:40. Obviously, it might not be feasible in practice to switch between systems within very short time intervals. This could be circumvented by adding a cost of switching a fuel system on, but this is regarded as outside the scope of this work. Yet, 20 minutes is believed to be sufficient time considering a sampling time of 10 seconds.

\section{Sensitivity to Disturbances}

The performance of the two solutions presented above will in this section be discussed with respect to sensitivity to noise. As noted previously, a system with feedback is typically more robust towards disturbances than with feedforward control. To evaluate this, input noise will be considered. In this work, the noise is assumed to be Gaussian white noise with a standard deviation of $5 \%$ of the maximum input signal.

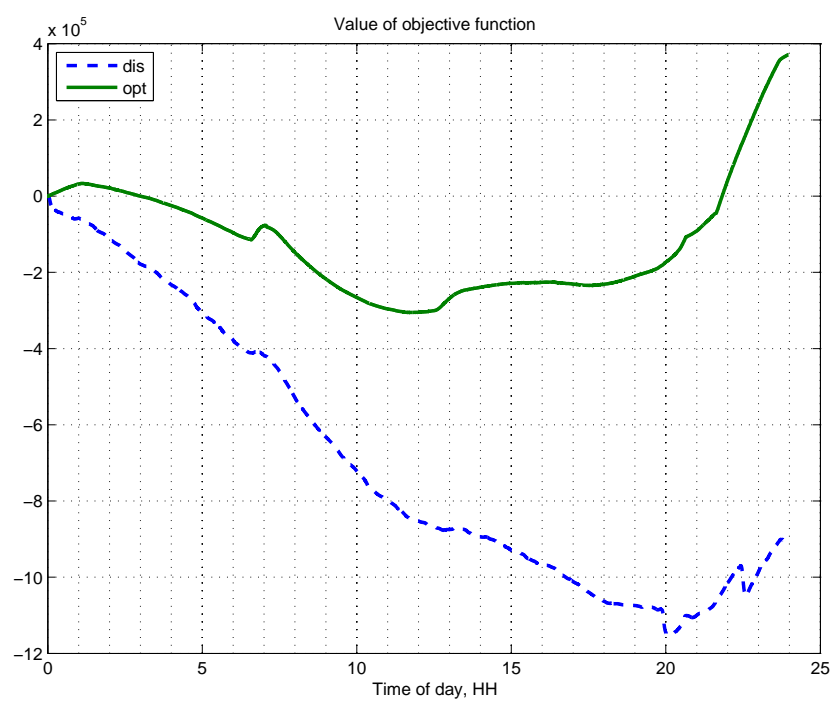

Fig. 9. The value of the objective function using the input obtained from the discrete and continuous optimization when input noise is present.

The value of the objective function during 24 hours with noise is depicted in Figure 9. As seen in the figure, the value is lower than without noise for both the discrete case and the optimal feedback/feedforward. However, the value of the objective function when using optimal feedback/feedforward is substantially larger than when using the discrete input. The reason for this is that the optimal feedback/feedforward tracks the reference better.

The tracking errors for the two input strategies are depicted in Figure 10. As seen in the figure, the error using the optimal feedback/feedforward is smaller than the error resulting from the discrete input. The mean tracking error is $3.40[M W]$ when using the optimal feedback/feedforward and $14.73[M W]$ when using the

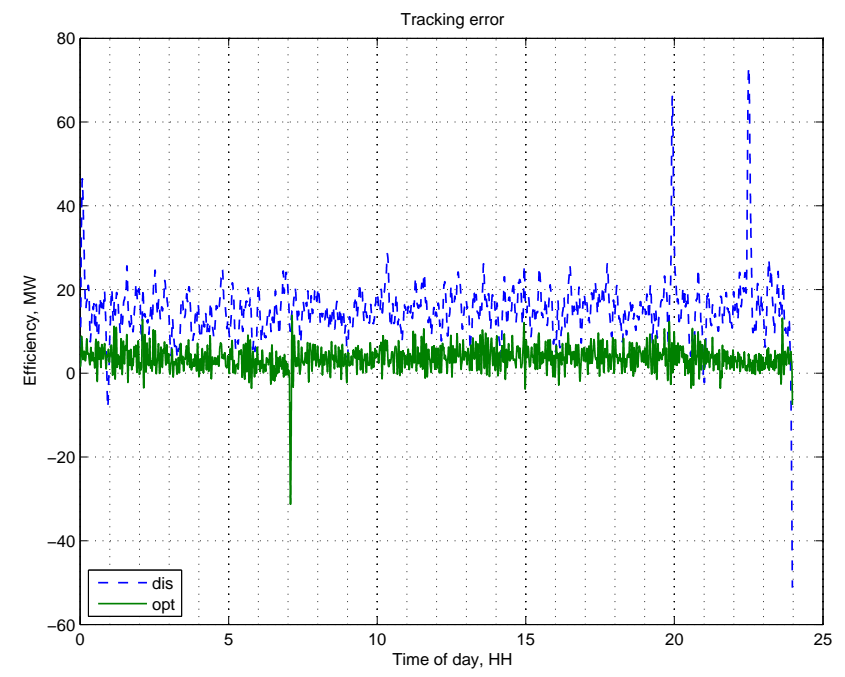

Fig. 10. The tracking error resulting from using the input obtained from the discrete and continuous optimization.

input from the discrete optimization. Furthermore, the standard deviation of the signal is also smaller using the feedback/feedforward solution as the values are $2.54[M W]$ and $6.54[M W]$ for feedback/feedforward and discrete, respectively.

The optimal feedback/feedforward is also superior in presence of noise with respect to the profit of the company. However, the difference it not as significant as when the tracking term is included. This can be observed in Figure 11 where the economical profit for the two solutions are depicted. The difference is about $161000[D K K]$; and thus, the gain of using the optimal feedback/feedforward is approximately $45 \%$.

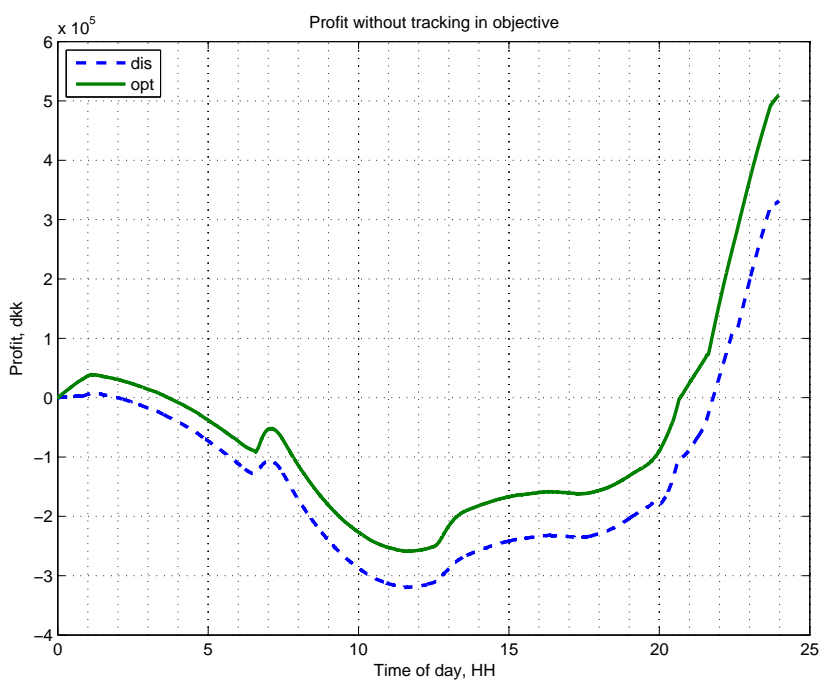

Fig. 11. The profit obtained during 24 hours when input noise is present.

\section{Discussion}

In this work, Pontryagin's maximum principle has been applied to a problem dealing with profit maxi- 
mization of a power plant. An optimal input strategy consisting of a combined feedback and feedforward has been developed such that profit is maximized over 24 hours of operation. The developed strategy is based on properties of the optimal control input and an initial solution of the adjoint equations obtained from discrete optimization. The two solutions, discrete input strategy and continuous feedback/feedforward, are evaluated both with and without input noise. As a result, the optimal feedback/feedforward yields a greater profit in both cases. In the presence of input noise, the feedback/feedforward solution yields a profit $45 \%$ larger than what is possible by using the discrete input strategy.

Future work in line with this paper would include improving the initial estimate of the switching function. In particular, a method using pseudo-spectral techniques to obtain a solution to the adjoint equation is proposed [18]. Using this method, it might be possible to obtain a sufficiently accurate estimate of the switching function $\boldsymbol{\sigma}(t)$ within less iterations of the algorithm suggested in this work. This could decrease the computational complexity and solving time, which makes the proposed method interesting for online implementation as a receding horizon.

\section{REFERENCES}

[1] Karl J. Åström and Bjorn Wittenmark. Computer-Controlled Systems - Theory and Design. Prentice-Hall International, 1990.

[2] David J. Bell and David H. Jacobson. Singular optimal control problems. Academic Press, London, 1975. Mathematics in Science and Engineering, Vol. 117.

[3] M. Bryds, P. D. Roberts, M. M. Badi, I. C. Kokkinos, and N. Abdullah. Double loop iterative strategies for hierarchical control of industrial processes. Automatica, 25(5):743-751, 1989.

[4] Arthur E. Bryson, Jr. and Yu Chi Ho. Applied optimal control. Hemisphere Publishing Corp. Washington, D. C., 1975. Optimization, estimation, and control, Revised printing.

[5] Lamberto Cesari. Optimization-theory and applications. Problems with ordinary differential equations, volume 17 of Applications of Mathematics (New York). Springer-Verlag, New York, 1983.

[6] Tongwen Chen and Bruce Francis. Optimal Sampled-Data Control Systems. Communication and Control Engineering Series, Springer-Verlag, 1995.

[7] Kristian Edlund, Tommy Mølbak, and Jan Dimon Bendtsen. Simple models for model-based portfolio load balancing controller synthesis. In Proceeding of IFAC Symposium on Power Plants and Power Systems, Tampere, Finland, 2009.

[8] Stein-Erik Fleten and Trine Krogh Kristoffersen. Short-term hydropower production planning by stochastic programming. Computers $\&$ Operations Research, 35(8):2656 - 2671, 2008.

[9] W. Kang, Q. Gong, I. M. Ross, and F. Fahroo. On the convergence of nonlinear optimal control using pseudospectral methods for feedback linearizable systems. Internatinal Journal of Robust and Nonlinear Control, 17:1251-1277, 2007.

[10] Martin Kragelund, Ulf Jönsson, John Leth, and Rafał Wisniewski. Optimal production planning of a power plant. In Proceedings of the International Conference on Control and Automation, Christchurch, 2009.

[11] Martin Kragelund, John Leth, and Rafael Wisniewski. Reference tracking and profit optimization of a power plant. In 1 st Virtual Control Conference (VCC 2010), 2010.

[12] Martin Kragelund, John Leth, and Rafał Wisniewski. Selecting actuator configuration for a benson boiler: Production economics. In Proceedings of the European Control Conference, Budapest, Hungary, 2009.
[13] Martin Kragelund, John Leth, and Rafał Wisniewski. Optimal usage of coal, gas, and oil in a power plant. IET Control Theory and Applications, 2010. Publiced in Special issue on Advances in Complex Control Systems Theory and Applications.

[14] Martin Kragelund, Rafał Wisniewski, Tommy Mølbak, René Just Nielsen, and Kristian Edlund. On propagating requirements and selecting fuels for a benson boiler. In Proceedings of the 17th IFAC World Congress, Seoul, South Korea, 2008.

[15] Faming Li, Mauricio de Oliveira, and Robert E. Skelton. Integrating control design and sensor/actuator selection. Proceedings of the 45th IEEE Conference on Decision \& Control, 2006.

[16] Mihajlo D. Mesarovic. Multilevel systems and concepts in process control. Proceedings of the IEEE, 58(1):111-125, January 1970.

[17] Magnus Olsson. On optimal hydropower bidding in systems with wind power : Modeling the impact of wind power on power markets. PhD thesis, KTH, Electric Power Systems, 2009.

[18] Anil V. Rao, David A. Benson, Christopher Darby, Michael A. Patterson, Camila Francolin, Ilyssa Sanders, and Geoffrey T. Huntington. Algorithm 902: GPOPS, a MATLAB software for solving multiple-phase optimal control problems using the Gauss pseudospectral method. ACM Trans. Math. Softw., 37(2):1-39, 2010.

[19] Atle Seierstad and Knut Sydsæter. Optimal Control Theory with Economic Applications. North Holland, 2007.

[20] Matt Thompson, Matt Davison, and Henning Rasmussen. Valuation and optimal operation of electric power plants in competitive markets. Operations Research, 52(4):546-562, 2004 .

[21] Richard Vinter. Optimal Control. Birkhäuser, 2000.

[22] E. Visser, B. Srinivasan, S. Palanki, and D Bonvin. A feedback-based implementation scheme for batch process optimization. Journal of Process Control, 10:399-410, 2000.

[23] Andrzej Wiśniewski. The continuous approximation of measurable mappings. Demonstratio Math., 29(3):529-531, 1996.

\section{APPENDIX I}

In this appendix, the optimal control input is found by examining the singular solution [2], [4], i.e., the equality

$$
\tilde{\boldsymbol{\sigma}}(t)=\boldsymbol{E}(t) \boldsymbol{B}^{T} \boldsymbol{\lambda}(t) \equiv \mathbf{0}
$$

is considered point-wise on a nondegenerate time interval. The matrix $\boldsymbol{E}(t)$ is constant in this time interval and will in the following be denoted by $\boldsymbol{E}$ to avoid confusion when taking the time derivative.

Some notation is introduced to simplify the equations throughout this appendix

$$
\begin{aligned}
\boldsymbol{R}(r, s, l) & =2 \boldsymbol{E} \boldsymbol{B}^{T} \boldsymbol{A}^{r T} \boldsymbol{Q}^{s} \boldsymbol{A}^{l} \\
\boldsymbol{D}(i, t) & =\sum_{n=0}^{i}(-1)^{n} \boldsymbol{R}(n, 0,0) \boldsymbol{q}^{(i-n)}(t),
\end{aligned}
$$

where $\boldsymbol{A}^{n T}=\prod_{j=1}^{n} \boldsymbol{A}^{T}$, with $\boldsymbol{A}^{0 T}=\boldsymbol{I}$ and $\boldsymbol{q}^{(j)}(t)$ is the $j$ th time derivative with $\boldsymbol{q}^{(0)}(t)=\boldsymbol{q}(t)$.

Differentiation of (18) yields

$$
\dot{\tilde{\boldsymbol{\sigma}}}(t)=\boldsymbol{E} \boldsymbol{B}^{T} \dot{\boldsymbol{\lambda}}(t)=\mathbf{0}
$$

Inserting the adjoint equation, (7), yields

$$
\begin{aligned}
\dot{\tilde{\boldsymbol{\sigma}}}(t) & =\boldsymbol{E} \boldsymbol{B}^{T}\left(2 \boldsymbol{Q} \boldsymbol{z}(t)-2 \boldsymbol{q}(t)-\boldsymbol{A}^{T} \boldsymbol{\lambda}(t)\right)=\mathbf{0} \Leftrightarrow \\
\boldsymbol{D}(0, t) & =\underbrace{\boldsymbol{R}(0,1,0)}_{\mathbf{0}} \boldsymbol{z}(t)-\frac{\boldsymbol{R}(1,0,0) \boldsymbol{\lambda}(t)}{2},
\end{aligned}
$$

where $\boldsymbol{R}(0,1,0)=\mathbf{0}$ follows from the structure of $\boldsymbol{Q}$, $\boldsymbol{B}$ and their sparsity, i.e., the relative degrees of the 
individual fuel systems are 3; and thus, the first and second time derivative will yield zero. Differentiating once more and inserting the adjoint equation yields the following equalities

$$
\begin{aligned}
\dot{\boldsymbol{D}}(0, t)= & -\frac{\boldsymbol{R}(1,0,0) \dot{\boldsymbol{\lambda}}(t)}{2} \\
\dot{\boldsymbol{D}}(0, t)= & -\boldsymbol{R}(1,0,0) \boldsymbol{Q} \boldsymbol{z}(t)+\boldsymbol{R}(1,0,0) \boldsymbol{q}(t) \\
& +\frac{\boldsymbol{R}(1,0,0) \boldsymbol{A}^{T} \boldsymbol{\lambda}(t)}{2} \\
\boldsymbol{D}(1, t)= & -\underbrace{\boldsymbol{R}(1,1,0)}_{\mathbf{0}} \boldsymbol{z}(t)+\frac{\boldsymbol{R}(2,0,0) \boldsymbol{\lambda}(t)}{2},
\end{aligned}
$$

where $\boldsymbol{R}(1,1,0)=\mathbf{0}$ follows from the structure of $\boldsymbol{Q}, \boldsymbol{B}$, and $\boldsymbol{A}$ as with $\boldsymbol{R}(0,1,0)=\mathbf{0}$ above. Now, it is possible to determine $\boldsymbol{\lambda}(t)$ using (18), (21), and (22). However, the objective is to find the optimal control input; therefore, (22) is differentiated once more which yields

$$
\begin{aligned}
\dot{\boldsymbol{D}}(1, t)= & \frac{\boldsymbol{R}(2,0,0) \dot{\boldsymbol{\lambda}}(t)}{2} \\
\dot{\boldsymbol{D}}(1, t)= & \boldsymbol{R}(2,0,0) \boldsymbol{Q} \boldsymbol{z}(t)-\boldsymbol{R}(2,0,0) \boldsymbol{q}(t) \\
& -\frac{\boldsymbol{R}(2,0,0) \boldsymbol{A}^{T} \boldsymbol{\lambda}(t)}{2} \\
\boldsymbol{D}(2, t)= & \boldsymbol{R}(2,1,0) \boldsymbol{z}(t)-\frac{\boldsymbol{R}(3,0,0) \boldsymbol{\lambda}(t)}{2}
\end{aligned}
$$

when the adjoint equations is inserted. As $\boldsymbol{R}(2,1,0) \neq \mathbf{0}$, (23) is differentiated again and the adjoint and system equations, (7) and (1), are inserted which yields

$$
\begin{aligned}
\dot{\boldsymbol{D}}(2, t)= & \boldsymbol{R}(2,1,0) \dot{\boldsymbol{z}}(t)-\frac{\boldsymbol{R}(3,0,0) \dot{\boldsymbol{\lambda}}(t)}{2} \\
\dot{\boldsymbol{D}}(2, t)= & \boldsymbol{R}(2,1,0)(\boldsymbol{A} \boldsymbol{z}(t)+\boldsymbol{B} \boldsymbol{u}(t)) \\
& -\frac{\boldsymbol{R}(3,0,0)\left(2 \boldsymbol{Q} \boldsymbol{z}(t)-2 \boldsymbol{q}(t)-\boldsymbol{A}^{T} \boldsymbol{\lambda}(t)\right)}{2} \\
\dot{\boldsymbol{D}}(2, t)= & \boldsymbol{R}(2,1,0)(\boldsymbol{A} \boldsymbol{z}(t)+\boldsymbol{B} \boldsymbol{u}(t)) \\
& -\boldsymbol{R}(3,0,0) \boldsymbol{Q} \boldsymbol{z}(t)+\boldsymbol{R}(3,0,0) \boldsymbol{q}(t) \\
& +\frac{\boldsymbol{R}(3,0,0) \boldsymbol{A}^{T} \boldsymbol{\lambda}(t)}{2} \\
\boldsymbol{D}(3, t)= & (\boldsymbol{R}(2,1,1)-\boldsymbol{R}(3,1,0)) \boldsymbol{z}(t) \\
& +\underbrace{\boldsymbol{R}(2,1,0) \boldsymbol{B}}_{\mathbf{0}} \boldsymbol{u}(t)+\frac{\boldsymbol{R}(4,0,0) \boldsymbol{\lambda}(t)}{2},
\end{aligned}
$$

where $\boldsymbol{R}(2,1,0) \boldsymbol{B}=\mathbf{0}$ is attributed the relative degree equals 3. Equation (24) is differentiated again which yields

$$
\begin{aligned}
\dot{\boldsymbol{D}}(3, t)= & (\boldsymbol{R}(2,1,1)-\boldsymbol{R}(3,1,0)) \dot{\boldsymbol{z}}(t) \\
& +\frac{\boldsymbol{R}(4,0,0) \dot{\boldsymbol{\lambda}}(t)}{2} \\
\dot{\boldsymbol{D}}(3, t)= & (\boldsymbol{R}(2,1,1)-\boldsymbol{R}(3,1,0))(\boldsymbol{A} \boldsymbol{z}(t)+\boldsymbol{B} \boldsymbol{u}(t)) \\
& +\frac{\boldsymbol{R}(4,0,0)\left(2 \boldsymbol{Q} \boldsymbol{z}(t)-2 \boldsymbol{q}(t)-\boldsymbol{A}^{T} \boldsymbol{\lambda}(t)\right)}{2} \\
\dot{\boldsymbol{D}}(3, t)= & (\boldsymbol{R}(2,1,2)-\boldsymbol{R}(3,1,1)) \boldsymbol{z}(t) \\
& +\underbrace{(\boldsymbol{R}(2,1,1)-\boldsymbol{R}(3,1,0)) \boldsymbol{B}}_{\mathbf{0}} \boldsymbol{u}(t) \\
& +\boldsymbol{R}(4,1,0) \boldsymbol{z}(t)-\boldsymbol{R}(4,0,0) \boldsymbol{q}(t) \\
& -\frac{\boldsymbol{R}(5,0,0) \boldsymbol{\lambda}(t)}{2} \\
\boldsymbol{D}(4, t)= & (\boldsymbol{R}(2,1,2)-\boldsymbol{R}(3,1,1)+\boldsymbol{R}(4,1,0)) \boldsymbol{z}(t) \\
& -\frac{\boldsymbol{R}(5,0,0) \boldsymbol{\lambda}(t)}{2},
\end{aligned}
$$

and $(\boldsymbol{R}(2,1,1)-\boldsymbol{R}(3,1,0)) \boldsymbol{B}=\mathbf{0}$ due to the relative degree of 3 . Using (23), (24), and (25), it is possible to calculate $\boldsymbol{z}(t)$. To obtain an expression for $\boldsymbol{u}(t)$, equation (25) is differentiated and adjoint and system equations are inserted

$$
\begin{aligned}
\dot{\boldsymbol{D}}(4, t)= & (\boldsymbol{R}(2,1,2)-\boldsymbol{R}(3,1,1)+\boldsymbol{R}(4,1,0)) \dot{\boldsymbol{z}}(t) \\
& -\frac{\boldsymbol{R}(5,0,0) \dot{\boldsymbol{\lambda}}(t)}{2} \\
\dot{\boldsymbol{D}}(4, t)= & (\boldsymbol{R}(2,1,2)-\boldsymbol{R}(3,1,1)+\boldsymbol{R}(4,1,0)) \boldsymbol{A} \boldsymbol{z}(t) \\
& +(\boldsymbol{R}(2,1,2)-\boldsymbol{R}(3,1,1)+\boldsymbol{R}(4,1,0)) \boldsymbol{B} \boldsymbol{u}(t) \\
& -\frac{\boldsymbol{R}(5,0,0)\left(2 \boldsymbol{Q} \boldsymbol{z}(t)-2 \boldsymbol{q}(t)-\boldsymbol{A}^{T} \boldsymbol{\lambda}(t)\right)}{2} \\
\dot{\boldsymbol{D}}(4, t)= & (\boldsymbol{R}(2,1,3)-\boldsymbol{R}(3,1,2)+\boldsymbol{R}(4,1,1)) \boldsymbol{z}(t) \\
& +(\boldsymbol{R}(2,1,2)-\boldsymbol{R}(3,1,1)+\boldsymbol{R}(4,1,0)) \boldsymbol{B} \boldsymbol{u}(t) \\
& -\boldsymbol{R}(5,0,0) \boldsymbol{Q} \boldsymbol{z}(t)+\boldsymbol{R}(5,0,0) \boldsymbol{q}(t) \\
& +\frac{\boldsymbol{R}(5,0,0) \boldsymbol{A}^{T} \boldsymbol{\lambda}(t)}{2} \\
\boldsymbol{D}(5, t)= & (\boldsymbol{R}(2,1,3)-\boldsymbol{R}(3,1,2) \\
& +\boldsymbol{R}(4,1,1)-\boldsymbol{R}(5,1,0)) \boldsymbol{z}(t) \\
& +(\boldsymbol{R}(2,1,2)-\boldsymbol{R}(3,1,1)+\boldsymbol{R}(4,1,0)) \boldsymbol{B} \boldsymbol{u}(t) \\
& +\frac{\boldsymbol{R}(6,0,0) \boldsymbol{\lambda}(t)}{2} \\
& +
\end{aligned}
$$

Using (26), it is possible to obtain an expression for $\boldsymbol{u}(t)$

$$
\boldsymbol{C}_{u} \boldsymbol{u}(t)=\boldsymbol{C}_{z} \boldsymbol{z}(t)+\boldsymbol{C}_{\tau}(t), \quad \boldsymbol{u}(t) \in \mathcal{U}(t)
$$

where

$$
\begin{aligned}
\boldsymbol{C}_{u} & =-(\boldsymbol{R}(2,1,2)-\boldsymbol{R}(3,1,1)+\boldsymbol{R}(4,1,0)) \boldsymbol{B} \\
\boldsymbol{C}_{z} & =\boldsymbol{R}(2,1,3)-\boldsymbol{R}(3,1,2)+\boldsymbol{R}(4,1,1)-\boldsymbol{R}(5,1,0) \\
\boldsymbol{C}_{\tau}(t) & =\frac{\boldsymbol{R}(6,0,0) \boldsymbol{\lambda}(t)}{2}-\boldsymbol{D}(5, t) .
\end{aligned}
$$

We remark that $\boldsymbol{C}_{u}$ can be used to verify that the generalized Legendre-Clebsch condition holds true in our case.

\section{Appendix II}

In this appendix, we will derive the lifting of the cost function, which is used in the discrete optimization. The 
term

$$
P(T)=\underbrace{\int_{0}^{T}-\boldsymbol{z}(t)^{T} \boldsymbol{Q} \boldsymbol{z}(t) d t}_{P_{2}(T)}+\underbrace{\int_{0}^{T} 2 \boldsymbol{q}(t)^{T} \boldsymbol{z}(t)+\varphi(t) d t}_{P_{1}(T)}
$$

is divided into two part during the discretization, i.e., the quadratic and affine terms are treated separately.

It is assumed that $\boldsymbol{q}(t)$ and $\varphi(t)$ can be approximated by piecewise constant functions for each time step, i.e.,

$$
\begin{aligned}
& \boldsymbol{q}(t)=\boldsymbol{q}_{k}, \quad k h<t<(k+1) h, \\
& \varphi(t)=\varphi_{k}, \quad k h<t<(k+1) h,
\end{aligned}
$$

where $h$ is the sampling time. Furthermore, the control is assumed piecewise constant as customary when digital to analogue conversion is performed using sample-hold circuits.

Using a fact from [1], the continuous time state $\boldsymbol{z}(t)$ in the dynamic system in (1) with constant input $\boldsymbol{u}_{0}$ can be described by

$$
\begin{aligned}
\boldsymbol{z}(t) & =e^{\boldsymbol{A} t} \boldsymbol{z}_{0}+\int_{0}^{t} e^{\boldsymbol{A}(t-s)} \boldsymbol{B} \boldsymbol{u}_{0}(s) d s \\
& =\left[\begin{array}{ll}
\boldsymbol{I} & \mathbf{0}
\end{array}\right] \exp \left\{\left[\begin{array}{cc}
\boldsymbol{A} & \boldsymbol{B} \\
\mathbf{0} & \mathbf{0}
\end{array}\right] t\right\}\left[\begin{array}{c}
\boldsymbol{z}_{0} \\
\boldsymbol{u}_{0}
\end{array}\right],
\end{aligned}
$$

where $\boldsymbol{I}$ is an identity matrix with appropriate dimension and $\boldsymbol{z}_{0}$ is the initial state. Using (30), it is possible to derive the following formula

$$
\begin{aligned}
\int_{0}^{h} e^{\boldsymbol{A} t} d t & =e^{\boldsymbol{A} h} \int_{0}^{h} e^{-\boldsymbol{A}(h-t)} d t \\
= & e^{\boldsymbol{A} h}\left(e^{-\boldsymbol{A} h} \cdot 0+\int_{0}^{h} e^{-\boldsymbol{A}(h-t)} \boldsymbol{I} d t\right) \\
= & e^{\boldsymbol{A} h}\left[\begin{array}{ll}
\boldsymbol{I} & \mathbf{0}
\end{array}\right] \exp \left\{\left[\begin{array}{cc}
-\boldsymbol{A} & \boldsymbol{I} \\
\mathbf{0} & \mathbf{0}
\end{array}\right] h\right\}\left[\begin{array}{l}
\mathbf{0} \\
\boldsymbol{I}
\end{array}\right] .
\end{aligned}
$$

The affine term is lifted by first using (30) and then (31)

$$
\begin{aligned}
& P_{1}(T)=\int_{0}^{T}\left(2 \boldsymbol{q}(t)^{T} \boldsymbol{z}(t)+\varphi(t)\right) d t \\
& =\sum_{k=0}^{N-1} 2 \boldsymbol{q}_{k}^{T} \int_{0}^{h}\left(e^{\boldsymbol{A} t} \boldsymbol{z}_{k}+\int_{0}^{t} e^{\boldsymbol{A}(t-s)} \boldsymbol{B} d s \boldsymbol{u}_{k}\right) d t \\
& +h \sum_{k=0}^{N-1} \varphi_{k} \\
& =\sum_{k=0}^{N-1} 2 \boldsymbol{q}_{k}^{T} \int_{0}^{h}\left[\begin{array}{ll}
\boldsymbol{I} & \mathbf{0}
\end{array}\right] e^{\tilde{\boldsymbol{A} t}}\left[\begin{array}{c}
\boldsymbol{z}_{k} \\
\boldsymbol{u}_{k}
\end{array}\right] d t+h \sum_{k=0}^{N-1} \varphi_{k} \\
& =\sum_{k=0}^{N-1} 2 \boldsymbol{q}_{k}^{T}\left[\begin{array}{ll}
\boldsymbol{I} & \mathbf{0}
\end{array}\right] e^{\tilde{\boldsymbol{A}} h}\left[\begin{array}{ll}
\boldsymbol{I} & \mathbf{0}
\end{array}\right] e^{\hat{\boldsymbol{A}} h}\left[\begin{array}{l}
\mathbf{0} \\
\boldsymbol{I}
\end{array}\right]\left[\begin{array}{l}
\boldsymbol{z}_{k} \\
\boldsymbol{u}_{k}
\end{array}\right] \\
& +h \sum_{k=0}^{N-1} \varphi_{k} \\
& =\sum_{k=0}^{N-1}\left(\boldsymbol{M}_{z_{k}} \boldsymbol{z}_{k}+\boldsymbol{M}_{u_{k}} \boldsymbol{u}_{k}+h \varphi_{k}\right),
\end{aligned}
$$

where

$$
\begin{aligned}
& \boldsymbol{M}_{z_{k}}=2 \boldsymbol{q}_{k}^{T}\left[\begin{array}{ll}
\boldsymbol{I} & \mathbf{0}
\end{array}\right] e^{\tilde{\boldsymbol{A}} h}\left[\begin{array}{ll}
\boldsymbol{I} & \mathbf{0}
\end{array}\right] e^{\hat{\boldsymbol{A}} h}\left[\begin{array}{l}
\mathbf{0} \\
\boldsymbol{I}
\end{array}\right]\left[\begin{array}{l}
\boldsymbol{I} \\
\mathbf{0}
\end{array}\right] \\
& \boldsymbol{M}_{u_{k}}=2 \boldsymbol{q}_{k}^{T}\left[\begin{array}{ll}
\boldsymbol{I} & \mathbf{0}
\end{array}\right] e^{\tilde{\boldsymbol{A}} h}\left[\begin{array}{ll}
\boldsymbol{I} & \mathbf{0}
\end{array}\right] e^{\hat{\boldsymbol{A}} h}\left[\begin{array}{l}
\mathbf{0} \\
\boldsymbol{I}
\end{array}\right]\left[\begin{array}{l}
\mathbf{0} \\
\boldsymbol{I}
\end{array}\right]
\end{aligned}
$$

with

$$
\hat{\boldsymbol{A}}=\left[\begin{array}{cc}
-\tilde{\boldsymbol{A}} & \boldsymbol{I} \\
\mathbf{0} & \mathbf{0}
\end{array}\right], \quad \tilde{\boldsymbol{A}}=\left[\begin{array}{cc}
\boldsymbol{A} & \boldsymbol{B} \\
\mathbf{0} & \mathbf{0}
\end{array}\right],
$$

and the matrices $\boldsymbol{I}$ and $\mathbf{0}$ are of appropriate dimensions. Next, the quadratic term is lifted by using (30)

$$
\begin{aligned}
& P_{2}(T)=\int_{0}^{T}-\boldsymbol{z}(t)^{T} \boldsymbol{Q} \boldsymbol{z}(t) d t \\
& =-\sum_{k=0}^{N-1} \int_{0}^{h}\left(\boldsymbol{z}_{k}^{T} e^{\boldsymbol{A}^{T} t}+\boldsymbol{u}_{k}^{T} \int_{0}^{t} \boldsymbol{B}^{T} e^{\boldsymbol{A}^{T}(t-s)} d s\right) \boldsymbol{Q} \\
& \left(e^{\boldsymbol{A} t} \boldsymbol{z}_{k}+\int_{0}^{t} e^{\boldsymbol{A}(t-s)} \boldsymbol{B} d s \boldsymbol{u}_{k}\right) d t \\
& =-\sum_{k=0}^{N-1} \int_{0}^{h}\left[\begin{array}{ll}
\boldsymbol{z}_{k}^{T} & \boldsymbol{u}_{k}^{T}
\end{array}\right] e^{\tilde{\boldsymbol{A}}^{T} t}\left[\begin{array}{l}
\boldsymbol{I} \\
\mathbf{0}
\end{array}\right] \boldsymbol{Q} \\
& {\left[\begin{array}{ll}
\boldsymbol{I} & \mathbf{0}
\end{array}\right] e^{\tilde{\boldsymbol{A}} t}\left[\begin{array}{l}
\boldsymbol{z}_{k} \\
\boldsymbol{u}_{k}
\end{array}\right] d t} \\
& =-\sum_{k=0}^{N-1}\left[\begin{array}{ll}
\boldsymbol{z}_{k}^{T} & \boldsymbol{u}_{k}^{T}
\end{array}\right] e^{\tilde{\boldsymbol{A}}^{T} h} \boldsymbol{Y}(h) e^{\tilde{\boldsymbol{A}} h}\left[\begin{array}{l}
\boldsymbol{z}_{k} \\
\boldsymbol{u}_{k}
\end{array}\right]
\end{aligned}
$$

where $\tilde{\boldsymbol{A}}$ is as above and

$$
\begin{aligned}
\boldsymbol{Y}(h) & =\int_{0}^{h} e^{-\tilde{\boldsymbol{A}}^{T}(h-t)} \overline{\boldsymbol{Q}} e^{-\tilde{\boldsymbol{A}}(h-t)} d t \\
\overline{\boldsymbol{Q}} & =\left[\begin{array}{l}
\boldsymbol{I} \\
\mathbf{0}
\end{array}\right] \boldsymbol{Q}\left[\begin{array}{ll}
\boldsymbol{I} & \mathbf{0}
\end{array}\right]
\end{aligned}
$$

The integral in (34) is the solution to a matrix differential equation

$$
\begin{gathered}
\boldsymbol{Y}(h)=\int_{0}^{h} e^{-\tilde{\boldsymbol{A}}^{T}(h-t)} \overline{\boldsymbol{Q}} e^{-\tilde{\boldsymbol{A}}(h-t)} d t \Rightarrow \\
-\frac{d}{d h} \boldsymbol{Y}(h)=\tilde{\boldsymbol{A}}^{T} \boldsymbol{Y}(h)+\boldsymbol{Y}(h) \tilde{\boldsymbol{A}}-\overline{\boldsymbol{Q}}, \quad \boldsymbol{Y}(0)=\mathbf{0} .
\end{gathered}
$$

Using the $\operatorname{Vec}(\cdot)$ notation which is defined as

$$
\operatorname{Vec}(\boldsymbol{P})=\left[\begin{array}{c}
p_{1} \\
\vdots \\
p_{n}
\end{array}\right]
$$

where $p_{i}$ is the columns of $\boldsymbol{P}$, it is possible to formulated $(35)$ as

$$
-\frac{d \operatorname{Vec}(\boldsymbol{Y}(h))}{d h}=\boldsymbol{F} \operatorname{Vec}(\boldsymbol{Y}(t))-\operatorname{Vec}(\overline{\boldsymbol{Q}})
$$

where

$$
\boldsymbol{F}=\left(\boldsymbol{I} \otimes \tilde{\boldsymbol{A}}^{T}+\tilde{\boldsymbol{A}}^{T} \otimes \boldsymbol{I}\right)
$$

and $\otimes$ denotes the Kronecker product. By using the solution to standard vector differential equation and (31), 
the solution to (37) is given by

$$
\begin{aligned}
\operatorname{Vec}(\boldsymbol{Y}(h)) & =\int_{0}^{h} e^{\boldsymbol{F}(h-\tau)} d \tau \operatorname{Vec}(\overline{\boldsymbol{Q}}) \\
& =e^{\boldsymbol{F} h}\left[\begin{array}{ll}
\boldsymbol{I} & \mathbf{0}
\end{array}\right] e^{\hat{\boldsymbol{F}} h}\left[\begin{array}{c}
\mathbf{0} \\
\boldsymbol{I}
\end{array}\right] \operatorname{Vec}(\overline{\boldsymbol{Q}}) \\
& =e^{\boldsymbol{F} h} \tilde{\boldsymbol{F}} \operatorname{Vec}(\overline{\boldsymbol{Q}}),
\end{aligned}
$$

where

$$
\tilde{\boldsymbol{F}}=\left[\begin{array}{ll}
\boldsymbol{I} & \mathbf{0}
\end{array}\right] e^{\hat{\boldsymbol{F}} h}\left[\begin{array}{l}
\mathbf{0} \\
\boldsymbol{I}
\end{array}\right], \quad \hat{\boldsymbol{F}}=\left[\begin{array}{cc}
\boldsymbol{F} & \boldsymbol{I} \\
\mathbf{0} & \mathbf{0}
\end{array}\right] .
$$

That is (33) can be expressed as

$$
P_{2}(T)=-\sum_{k=0}^{N-1}\left[\begin{array}{ll}
\boldsymbol{z}_{k}^{T} & \boldsymbol{u}_{k}^{T}
\end{array}\right]\left[\begin{array}{ll}
\boldsymbol{N}_{z z} & \boldsymbol{N}_{z u} \\
\boldsymbol{N}_{u z} & \boldsymbol{N}_{u u}
\end{array}\right]\left[\begin{array}{l}
\boldsymbol{z}_{k} \\
\boldsymbol{u}_{k}
\end{array}\right]
$$

where

$$
\begin{aligned}
& \boldsymbol{N}_{z z}=\left[\begin{array}{ll}
\boldsymbol{I} & \mathbf{0}
\end{array}\right] e^{\tilde{\boldsymbol{A}}^{T} h} \operatorname{Vec}^{-1}\left(e^{\boldsymbol{F} h} \tilde{\boldsymbol{F}} \operatorname{Vec}(\overline{\boldsymbol{Q}})\right) e^{\tilde{\boldsymbol{A}} h}\left[\begin{array}{l}
\boldsymbol{I} \\
\mathbf{0}
\end{array}\right] \\
& \boldsymbol{N}_{z u}=\left[\begin{array}{ll}
\boldsymbol{I} & \mathbf{0}
\end{array}\right] e^{\tilde{\boldsymbol{A}}^{T} h} \operatorname{Vec}^{-1}\left(e^{\boldsymbol{F} h} \tilde{\boldsymbol{F}} \operatorname{Vec}(\overline{\boldsymbol{Q}})\right) e^{\tilde{\boldsymbol{A}} h}\left[\begin{array}{l}
\mathbf{0} \\
\boldsymbol{I}
\end{array}\right] \\
& \boldsymbol{N}_{u z}=\left[\begin{array}{ll}
\mathbf{0} & \boldsymbol{I}
\end{array}\right] e^{\tilde{\boldsymbol{A}}^{T} h} \operatorname{Vec}^{-1}\left(e^{\boldsymbol{F} h} \tilde{\boldsymbol{F}} \operatorname{Vec}(\overline{\boldsymbol{Q}})\right) e^{\tilde{\boldsymbol{A}} h}\left[\begin{array}{l}
\boldsymbol{I} \\
\mathbf{0}
\end{array}\right] \\
& \boldsymbol{N}_{u u}=\left[\begin{array}{ll}
\mathbf{0} & \boldsymbol{I}
\end{array}\right] e^{\tilde{\boldsymbol{A}}^{T} h} \operatorname{Vec}^{-1}\left(e^{\boldsymbol{F} h} \tilde{\boldsymbol{F}} \operatorname{Vec}(\overline{\boldsymbol{Q}})\right) e^{\tilde{\boldsymbol{A}} h}\left[\begin{array}{l}
\mathbf{0} \\
\boldsymbol{I}
\end{array}\right]
\end{aligned}
$$

with $\mathrm{Vec}^{-1}(\cdot)$ denoting the inverse of the Vec-operator in $(36)$, i.e., reshaping the vector into a matrix. 